\title{
ANATOMICAL AND FUNCTIONAL CONNECTIONS BETWEEN THE LOCUS COERULEUS AND THE NUCLEUS TRACTUS SOLITARIUS IN NEONATAL RATS
}

\author{
L. T. LOPES, ${ }^{a}$ L. G. A. PATRONE, ${ }^{a}$ K.-Y. LI, ${ }^{b}$ \\ A. N. IMBER, ${ }^{b}$ C. D. GRAHAM, \\ L. H. GARGAGLIONI ${ }^{a}$ AND R. W. PUTNAM ${ }^{\text {b* }}$ \\ ${ }^{a}$ Dept of Animal Morphology and Physiology. São Paulo \\ State University, FCAV, Jaboticabal, SP, Brazil \\ ${ }^{\mathrm{b}}$ Dept of Neuroscience, Cell Biology and Physiology, Wright State \\ University, Dayton, $\mathrm{OH}, \mathrm{USA}$
}

\begin{abstract}
This study was designed to investigate brain connections among chemosensitive areas in newborn rats. Rhodamine beads were injected unilaterally into the locus coeruleus (LC) or into the caudal part of the nucleus tractus solitarius (cNTS) in Sprague-Dawley rat pups (P7-P10). Rhodamine-labeled neurons were patched in brainstem slices to study their electrophysiological responses to hypercapnia and to determine if chemosensitive neurons are communicating between LC and cNTS regions. After 7-10 days, retrograde labeling was observed in numerous areas of the brainstem, including many chemosensitive regions, such as the contralateral LC, cNTS and medullary raphe. Whole-cell patch clamp was done in cNTS. In 4 of 5 retrogradely labeled cNTS neurons that projected to the LC, firing rate increased in response to hypercapnic acidosis $\left(15 \% \mathrm{CO}_{2}\right)$, even in synaptic blockade medium (SNB) (high $\mathrm{Mg}^{2+} /$ low $\mathrm{Ca}^{2+}$ ). In contrast, 2 of 3 retrogradely labeled LC neurons that projected to cNTS had reduced firing rate in response to hypercapnic acidosis, both in the presence and absence of SNB.
\end{abstract}

\footnotetext{
${ }^{*}$ Corresponding author. Address: Dept of Neuroscience, Cell Biology and Physiology, Wright State University, 3640 Colonel Glenn Highway, Dayton, OH 45435, USA. Tel: + 1-937-775-2288; fax: +1-937775-3391.

E-mail address: robert.putnam@wright.edu (R. W. Putnam).

Abbreviations: aCSF, artificial cerebral spinal fluid; Amb, ambiguus nucleus; AP, area postrema; Bötz, Bötzinger complex; Cereb, cerebellum; Cl, chemosensitivity index; CLC, contralateral LC; cNTS, caudal nucleus tractus solitarius; CVL, caudoventrolateral reticular nucleus; dHyp, dorsomedial hypothalamus; dIPAG, periaqueductal gray dorsolateral portion; DR, dorsal raphe; EGTA, ethylene glycol tetraacetic acid; HEPES, 4-(2-hydroxyethyl)-1-piperazineethanesulfo nic acid; iNTS, intermediate nucleus tractus solitarius; IO, inferior olive; KF, Kölliker-Fuse; LC, locus coeruleus; IHyp, lateral hypothalamus; IPAG, periaqueductal gray lateral portion; LRtPC/LRt, lateral reticular nucleus, parvicellular part/lateral reticular nucleus; LY, Lucifer Yellow; MR, medullary raphe; NTS, nucleus tractus solitarius; PAG, periaqueductal gray; $\mathrm{PB}$, parabrachial nucleus; PBS, phosphatebuffered saline; pHyp, posterior hypothalamus; Pre-Bötz, PreBötzinger complex; Py, pyramidal tract; RTN, retrotrapezoid nucleus; RVL, rostroventrolateral reticular nucleus; SNB, synaptic blockade medium; sp5, spinal trigeminal tract; SpVe/Mve, spinal vestibular nucleus/medial vestibular nucleus; SubC, subcoeruleus region; SubCA, subcoeruleus nucleus alpha portion; SubCD, subcoeruleus nucleus dorsal portion; SubCV, subcoeruleus nucleus ventral portion; vIPAG, periaqueductal gray ventrolateral portion.
}

Extensive anatomical connections among chemosensitive brainstem regions in newborn rats were found and at least for the LC and cNTS, the connections involve some $\mathrm{CO}_{2}$-sensitive neurons. Such anatomical and functional coupling suggests a complex central respiratory control network, such as seen in adult rats, is already largely present in neonatal rats by at least day P7-P10. Since the NTS and the LC play a major role in memory consolidation, our results may also contribute to the understanding of the development of memory consolidation. (C) 2016 IBRO. Published by Elsevier Ltd. All rights reserved.

Key words: chemosensitivity, development, electrophysiology, hypercapnia, retrograde labeling, memory consolidation.

\section{INTRODUCTION}

The respiratory system is not mature at birth, but undergoes significant postnatal development (Mortola, 2001). A critical period exists around postnatal day (P) 12-13, when an imbalance between heightened inhibition and suppressed excitation is evident both neurochemically as well as electrophysiologically (Wong-Riley et al., 2013). Likewise, the ventilatory response to hypercapnia changes as a function of neonatal development (Putnam et al., 2005). The $\mathrm{CO}_{2}$ response is vigorous in the first postnatal days (P1-P5), but then decreases and reaches its lowest point at P8. Subsequently, the response rises until P21, when it seems to achieve the adult level (Stunden et al., 2001; Putnam et al., 2005; Greer, 2012).

There is growing evidence suggesting that respiratory central chemoreception is a distributed property including multiple brainstem regions and neuronal types that may change over development (Coates et al., 1993; Nattie, 1999; Nattie and Li, 2002, 2009; Putnam et al., 2004; Richerson, 2004; Biancardi et al., 2008; Gargaglioni et al., 2010; Hodges and Richerson, 2010; Putnam, 2010). Among these chemosensitive sites are the locus coeruleus (LC) (Elam et al., 1981; Pineda and Aghajanian, 1997; Filosa et al., 2002; Gargaglioni et al., 2010) and the caudal region of the nucleus tractus solitarius (cNTS) (Dean et al., 1989, 1990; Nattie and Li, 2002; Conrad et al., 2009; Dean and Putnam, 2010). A high percentage $(>80 \%)$ of LC neurons respond to elevated $\mathrm{CO}_{2} / \mathrm{H}^{+}$with an increased firing rate (Elam et al., 1981; 
Pineda and Aghajanian, 1997; Filosa et al., 2002), focal acidification of the LC increases ventilation (Coates et al., 1993) and lesioning of a substantial portion of the LC reduces the ventilatory response to inspired $\mathrm{CO}_{2}(\mathrm{Li}$ and Nattie, 2006; Biancardi et al., 2008). Interestingly, there is a marked developmental change in the intrinsic chemosensitivity of LC neurons. In LC neurons from neonates younger than $\mathrm{P} 10$, a high percentage of neurons are $\mathrm{CO}_{2}$ sensitive (70-80\%) and the chemosensitivity index $(\mathrm{Cl})$, a measure of the magnitude of the neuronal chemosensitive response, is about 235\%; whereas in LC neurons from older neonates ( $>\mathrm{P} 10$ ), a high percentage of neurons are also activated by hypercapnia, but the magnitude of the response is markedly lower $(\mathrm{Cl}$ of $125 \%$ ) (Hartzler et al., 2008; Nichols et al., 2008; Gargaglioni et al., 2010). Compared to other regions, most notably the retrotrapezoid nucleus (RTN) where the $\mathrm{Cl}$ is very high at $300 \%$ (determined at $10 \% \mathrm{CO}_{2}$; Putnam et al., 2004), the $\mathrm{Cl}$ of LC neurons from young neonates is fairly large 180$220 \%$ (measured at $7.5 \%, 10 \%$ and $15 \% \mathrm{CO}_{2}$; Putnam, unpublished data) while it is very low in LC neurons from older neonates (115-135\% measured at $7.5 \%, 10 \%$ and $15 \% \mathrm{CO}_{2}$; Putnam, unpublished data). These studies indicate that around 10 days after birth, LC neurons undergo a major reduction in chemosensitivity with the percentage of intrinsically chemosensitive neurons and the magnitude of their response both decreasing dramatically.

As to the NTS, this receives inputs from peripheral chemoreceptors and lung stretch receptors (Donoghue et al., 1984; Bonham and McCrimmon, 1990; Mifflin, 1992; Koshiya and Guyenet, 1996) and it is suggested to be an important site of ventilatory and cardiovascular control (Barraco et al., 1990; Mifflin, 1992). About $40-50 \%$ of NTS neurons depolarize and/or increase their firing rate upon exposure to hypercapnia (at levels of $7 \%$, $10 \%$ and $15 \% \mathrm{CO}_{2}$ (Dean et al., 1989, 1990; Huang et al., 1997; Mulkey et al., 2004), the magnitude of this response, as determined by the $\mathrm{Cl}$, is about half that of neurons from the RTN, or about 155-175\% (measured at $15 \% \mathrm{CO}_{2}$; Conrad et al., 2009), and the chemosensitive response of these neurons does not change with development (Conrad et al., 2009; Nichols et al., 2009). Further, ventilation is increased when the NTS-especially the caudal part (cNTS) near the area postrema (AP)-is focally acidified (Coates et al., 1993; Nattie, 2001; Nattie and $\mathrm{Li}, 2002)$ and chemical lesioning or synaptic blockade of the NTS causes a reduction in basal ventilation and in respiratory chemosensitivity to $\mathrm{CO}_{2}$ (Berger and Cooney, 1982; Nattie and Li, 2002, 2008; although see Favero et al., 2011). These studies strongly suggest that the NTS, and especially the cNTS, is involved in central chemosensitivity and central ventilatory control (Dean and Putnam, 2010).

Although much is known about these nuclei, central chemosensitivity appears to involve a complex network that includes neurons from the LC and cNTS, as well as many other brainstem areas. This network is poorly understood, including the roles of the various areas, the interconnections and the functional communication between these areas. Also, almost all previous studies on the nature of the network have involved studies of adults and thus very little is known about the development of the central respiratory network.

It is noteworthy that the neuronal pathways we are studying in neonates play significant roles in functions others than the control of breathing. Considerable evidence has accumulated to show that highly emotional events help in the consolidation of memories (McGaugh, 2000). It appears that epinephrine or other stressrelated chemicals enhance memory consolidation in part through activating NTS neurons which ultimately results in ß-adrenergic activation of the basolateral amygdala (Roozendaal et al., 1999; Clayton and Williams, 2000; Garcia-Medina and Miranda, 2013; Mello-Carpes and Izquierdo, 2013). One pathway of excitation from the NTS to the amygdala that has been proposed involves projections from the NTS, to the Paragigantocellularis nucleus, to the LC, and ultimately to the amygdala (Clayton and Williams, 2000; Mello-Carpes and Izquierdo, 2013), although the possibility of direct neuronal connections between the NTS and the LC (Van Bockstaele et al., 1999; Clayton and Williams, 2000) and the NTS and the basolateral amygdala have been suggested (Roozendaal et al., 1999). These various pathways have been shown to be especially critical to the consolidation of memories associated with object recognition (Mello-Carpes and Izquierdo, 2013) and aversive conditions (Clayton and Williams, 2000). There has been little study of the development of this memory consolidation pathway. Nirogi et al. (2012) showed that the consolidation of object recognition memories was markedly less in juvenile vs. adult rats. They showed that the levels of norepinephrine were lower in juvenile vs. adult rats but that the object recognition performance of juvenile rats could be improved with yohimbine, an $\alpha$ adrenergic receptor antagonist (Nirogi et al., 2012). These data suggest that the anatomical pathway for memory consolidation is intact in juveniles. Our current anatomical studies with neonates are likely to contribute to our understanding of the development of these important memory consolidation pathways, especially given our focus on the projections associated with NTS and LC neurons.

Therefore, in this study, we have focused on the LC and the cNTS of neonatal rats, using retrograde labeling to identify possible neural connections between these two areas and other known chemosensitive regions. We further used whole-cell and loose patch clamping of retrogradely labeled neurons to test the firing rate response of neurons to hypercapnia in order to verify whether neurons projecting from LC to cNTS and from cNTS to LC could carry chemosensitive information.

\section{EXPERIMENTAL PROCEDURES}

\section{Animals}

Mixed sex neonatal Sprague-Dawley rats, age between P7 and P10, were used for microinjection surgeries. The mothers had free access to water and food and were housed in a temperature-controlled room at $24-26^{\circ} \mathrm{C}$, with a 12:12-h light:dark cycle (lights on at 7:00 AM). The pups stayed together with their mother during all 
pre- and post-operative periods. All experiments were done between $8 \mathrm{am}$ and $5 \mathrm{pm}$. The study was in compliance with the guidelines of and it was approved by the Wright State University Institutional Animal Care and Use Committee. Wright State University is accredited by AAALAC and is covered by $\mathrm{NIH}$ Assurance (no. A3632-01).

\section{Surgery}

For LC surgeries, the surgical procedures were performed under anesthesia with isofluorane $(5 \%$ inhaled to induce anesthesia and $2 \%$ inhaled to maintain anesthesia). For cNTS surgeries, the rats were anesthetized with ketamine $(75 \mathrm{mg} / \mathrm{kg}$ I.P. $)+$ xylasine $(5 \mathrm{mg} / \mathrm{kg}) \quad(0.1 \mathrm{~mL} / 20 \mathrm{~g})$. Subcutaneous bupivicaine $(0.15 \mathrm{~mL})$ was given for the local anesthesia, and subcutaneous injections of the analgesic agent carprofen $(0.5 \mathrm{mg} / \mathrm{ml}$; vol: $0.1 \mathrm{~mL} / 10 \mathrm{~g})$ were made before and $12 \mathrm{~h}$ after the surgical procedure.

For microinjections of rhodamine beads, the pup was fixed to a stereotaxic frame (David Kopf, model 900, Tujunga, CA, USA adapted for neonates) and the skin was sterilized with povidine solution. Then specific coordinates were used to locate the LC (anteroposterior $=-3.7 \mathrm{~mm}$; lateral $=-1.1 \mathrm{~mm}$ from sagittal suture; dorso-ventral $=-4.0 \mathrm{~mm}$ deep from the skull). For cNTS location, the animal's head was angled $45^{\circ}$ (nose directed downward), an incision with a scalpel in the neck region was done followed by removing a piece of the occipital bone as well as the membranes covering the NTS region, making it possible to view the calamus scriptorius, used as reference for the coordinates (lateral $=-0.1 \mathrm{~mm}$; dorso-ventral $=-0.3 \mathrm{~mm}$ ). Coordinates were based on a stereotaxic atlas for rats (Paxinos and Watson, 1997). A dental injection needle (Mizzy, $200 \mu \mathrm{m}$ $\mathrm{OD}$ ) connected to a $1-\mu \mathrm{L}$ Hamilton syringe by $\mathrm{PE}-10$ catheter was inserted into the LC or CNTS for $70 \mu \mathrm{L}$ or $50 \mu \mathrm{L}$ microinjection of rhodamine beads solution, respectively (Lumafluor, Inc. red retrobeads, St Louis, MO, USA) and $5 \mathrm{~min}$ were allowed to prevent leakage of rhodamine beads before the injection needle was removed. All injections were performed manually and the microinjection volume was chosen based on pilot experiments. At the end of the surgical procedure, the animal's skin was held with surgical glue (Vetbond, Tissue Adhesive) and cleaned with povidine solution.

\section{Slice preparation}

Between 7 to 10 days after the injection of the fluorescent dye, the labeled rats were then anesthetized with $100 \%$ $\mathrm{CO}_{2}$ and rapidly decapitated. The brain was removed and placed in $4-6^{\circ} \mathrm{C}$ artificial cerebral spinal fluid (aCSF) solution equilibrated in $95 \% \mathrm{O}_{2} / 5 \% \mathrm{CO}_{2}$ (see Solutions for composition). Coronal slices $(\sim 300 \mu \mathrm{m})$, extending rostrally from the cNTS (near the AP) to the hypothalamus were prepared using a PelcoVibratome 1000. Newly prepared slices were either rapidly transferred into $4 \%$ paraformaldehyde solution (see Solutions) and fixed for 3 days in the refrigerator at $4{ }^{\circ} \mathrm{C}$ for anatomy studies or allowed to equilibrate for $\sim 1 \mathrm{hr}$ at room temperature in aCSF equilibrated with $95 \%$ $\mathrm{O}_{2} / 5 \% \mathrm{CO}_{2}$ for electrophysiology studies.

\section{Anatomy}

After fixation, slices to be used for anatomy studies were washed in phosphate-buffered saline (PBS, see Solutions) and placed in a Plexiglas chamber on the stage of a Nikon Eclipse Ti-S Inverted Light Microscope. The slice was bathed in a PBS solution and held in place with a nylon grid. Brightfield illumination was used to identify the edges of the slice; some references, such as the central canal (CC), fourth ventricle and cerebellum (Cereb), were used to identify the rostrocaudal level and their position in relation to bregma and also identify various regions within the slice (based on landmarks from Paxinos and Watson, 1997). The same region was then studied using epifluorescence. Rhodamine-labeled neurons were excited at $540-580 \mathrm{~nm}$ and emitted light collected at $600-660 \mathrm{~nm}$ using a Nikon Y-2 E/C fluorescence filter and a 75-W xenon arc lamp. Emitted fluorescence was visualized using a CoolSNAP HQ2 digital camera (Photometrics) and Metafluor 4.6r5 software (Universal Imaging) and the fluorescence images saved to a Gateway 2000 E-3100 computer. Retrogradely labeled slices were first examined at lower objective $(4 \times)$ and rhodamine-labeled terminal fields were identified. The regions in which these fields occurred were verified more accurately using brightfield microscopy, supported by anatomical markers from Paxinos and Watson (1997). Such rhodamine-labeled terminal fields were found in many regions of the brain (Figs. 1 and 2). The degree of labeling was estimated more precisely by studying these rhodamine-labeled fields at higher objective $(10 \times$ for labeling drawings with red dots and in some instances $20 \times$ to verify that the labeling was in fact rhodamine-labeled neurons) using fluorescence microscopy to estimate the number of rhodamine-labeled neuronal cell bodies.

All slices of all animals, from cNTS to the hypothalamus were examined on the microscope by only one trained person for retrograde labeling location. All rhodamine fluorescence observed in each slice's region was immediately and carefully marked on schematic drawings made from Paxinos and Watson (1997) in order to preserve the anatomical locations of retrograde labeling within the brain.

\section{Electrophysiology}

Slices to be used in electrophysiological studies were placed in a superfusion chamber and held in place with a nylon grid. Slices were superfused at a rate of $\sim 3 \mathrm{~mL} / \mathrm{min}$ with aCSF or SNB (synaptic blockade medium) (see Solutions) solution at $\sim 35^{\circ} \mathrm{C}$. Glass electrodes were made from thin-walled borosilicate glass (outer diameter $1.5 \mathrm{~mm}$, inner diameter $1.12 \mathrm{~mm}$ ) pulled to a tip resistance of $3-5 \mathrm{M} \Omega$ with a Narishige Model PC-10 pipette puller. The bath was grounded with an $\mathrm{Ag}-\mathrm{AgCl}_{2}$ wire. Pipettes were filled with pipette filling solution (see Solutions). 


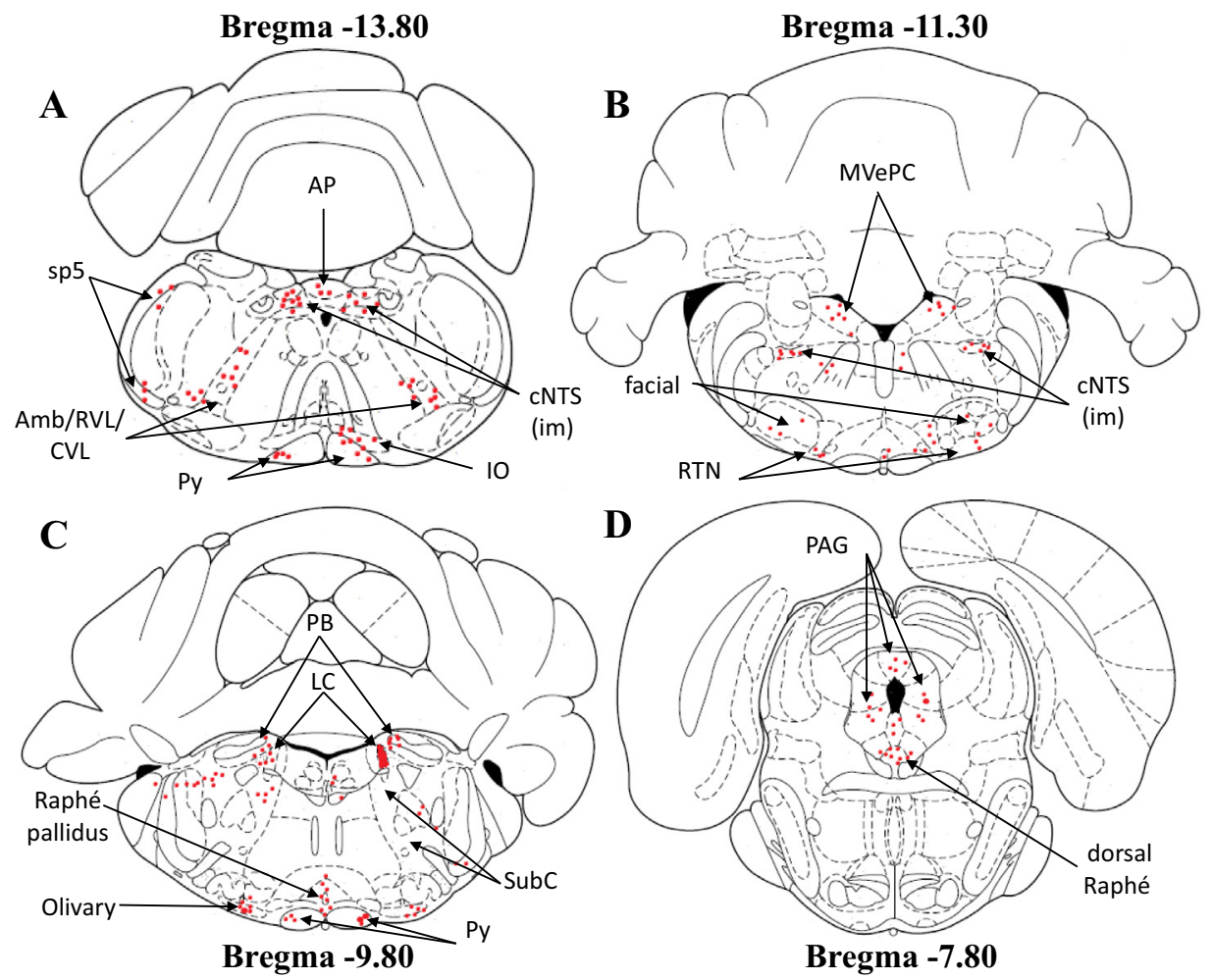

Fig. 1. Schematic drawings of four different slices showing the location of retrogradely labeled neurons (red dots) in different brain regions from neonatal rats injected with rhodamine beads into the right side of the LC. Note that the microinjection site of rhodamine beads is represented in schema 3C on LC right side. The schematic drawings are taken from Paxinos and Watson (1997). Each red dot represents a distinct neuron loaded with fluorescent rhodamine. Amb/RVL/CVL, ambiguus nucleus/rostroventrolateral reticular nucleus/caudoventrolateral reticular nucleus; AP, area postrema; cNTS, caudal nucleus tractus solitarius; iNTS, intermediate nucleus tractus solitarius; IO, inferior olive; LC, locus coeruleus; MVePC, medial vestibular nucleus, parvicellular part; PAG, periaqueductal gray; PB, parabrachial nucleus; Py, pyramidal tract; RTN, retrotrapezoid nucleus; SubC, subcoeruleus; sp5, spinal trigeminal tract.

LC and CNTS neurons were visualized using an upright microscope (Nikon FN1) with a $60 \times$ waterimmersion objective. Neurons retrogradely labeled with rhodamine beads were visualized using identical excitation/emission wavelengths as described above (see Anatomy). Using NIS Elements AR 3.0 software (Nikon), rhodamine fluorescence was matched to healthy neurons visualized without fluorescence and these neurons were targeted for patching. Electrophysiological recordings were done using an Axopatch 200B amplifier and analyzed using PCLAMP software version 10 . The pipette was brought to the surface of a neuron under positive pressure to prevent the tip from plugging and then a gentle suction was applied to the pipette until a $\sim 1 \mathrm{G} \Omega$ seal was obtained.

For cNTS neurons, after the $G \Omega$ seal was established, a rapid negative pressure was applied to establish a whole-cell configuration. Such neurons were current clamped and membrane potential was measured. These neurons showed the intracellular voltage changes associated with action potentials and could be used to determine the firing rate as well. In LC neurons, the cellattached patch mode was used to record spontaneous firing at a holding potential of $0 \mathrm{mV}$ under voltage clamp. The $G \Omega$ seal in the cell-attached patch gave a sufficient signal-noise ratio that extracellular currents were clearly visible and thus firing rate could be determined from these currents.
For all neurons, recordings began when an initial stable firing rate was obtained and the effect of hypercapnia on firing rate was studied by switching the perfusate (standard aCSF or SNB solution) bubbled with $5 \% \mathrm{CO}_{2}$ to the same perfusate bubbled with $15 \% \mathrm{CO}_{2}$. We chose to use this high level of hypercapnia $(15 \%$ $\mathrm{CO}_{2}$ ) since our primary aim in these initial electrophysiology studies was to determine whether neurons were chemosensitive or not. We have previously shown with cNTS neurons that about $40-50 \%$ of cNTS neurons from neonatal rats will not change their firing rate in response to this level of hypercapnia (non-chemosensitive neurons) and about $40-50 \%$ will increase their firing rate in response to hypercapnia (chemosensitive neurons), while the firing rate in the remaining neurons is reduced upon exposure to $15 \% \mathrm{CO}_{2}$ (Conrad et al., 2009). Similarly, $15 \% \mathrm{CO}_{2}$ results in an increased firing rate in $70-80 \%$ of LC neurons from neonatal rats with the remainder not responding to hypercapnia (Li and Putnam, 2013). Both cNTS and LC neurons also respond with increased firing rate to hypercapnia of $10 \%$ and $7-8 \%$ (Dean et al., 1990; Oyamada et al., 1998; Li and Putnam, 2013) and LC neurons respond with a decreased firing rate in response to hypocapnia $\left(2.5 \% \mathrm{CO}_{2}\right)$ ( $\mathrm{Li}$ and Putnam, 2013). Thus the electrophysiology experiments as designed here allow for the differentiation of whether a neuron is chemosensitive or not. 

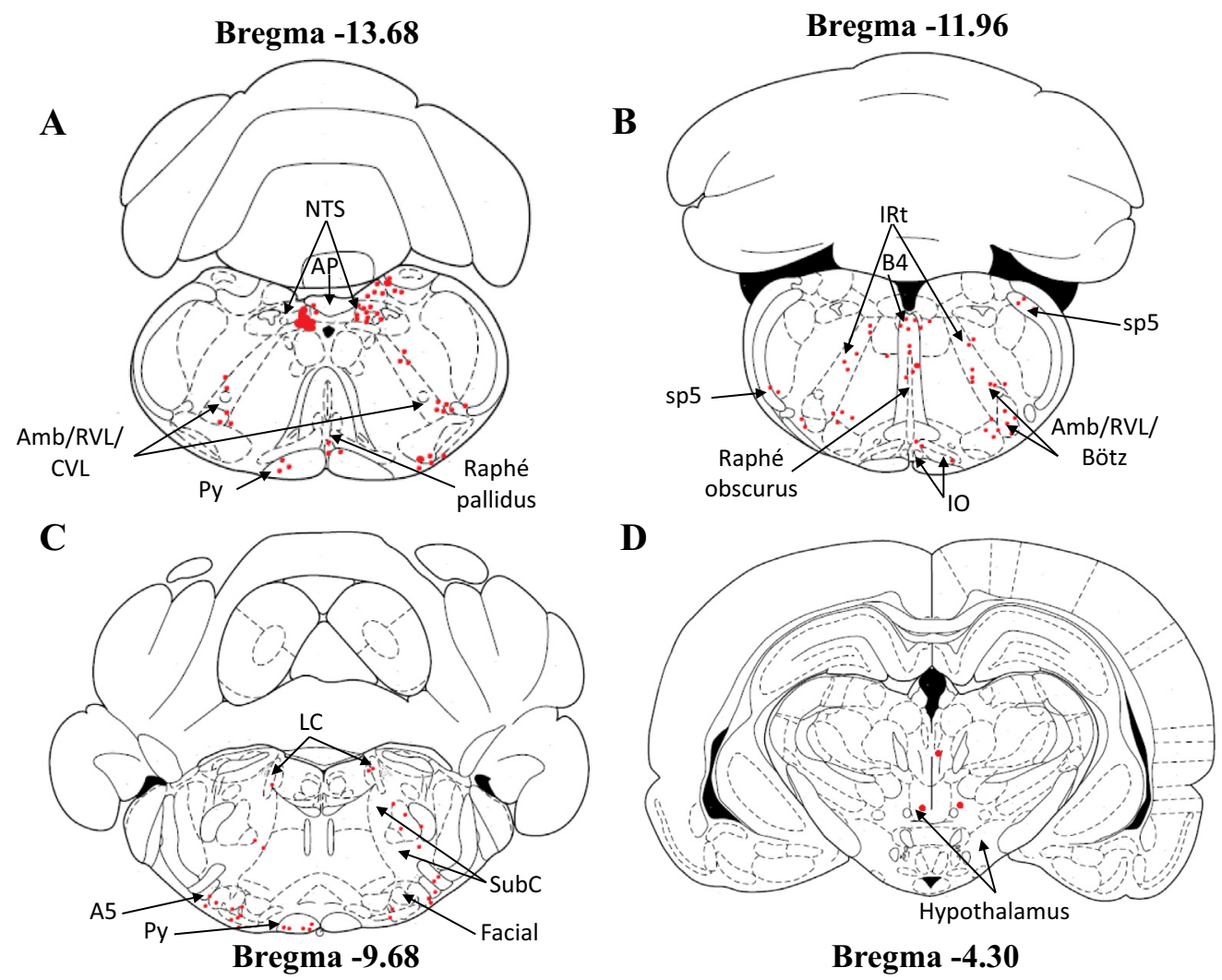

Fig. 2. Schematic drawings of four different slices showing the location of retrogradely labeled neurons (red dots) in different brain regions from neonatal rats injected with rhodamine beads into cNTS. Note that the microinjection site of rhodamine beads is represented in schema 4A. The schematic drawings are taken from Paxinos and Watson (1997). Each red dot represents a distinct neuron loaded with fluorescent rhodamine. Amb/ RVL/CVL, ambiguus nucleus/rostroventrolateral reticular nucleus/caudoventrolateral reticular nucleus; AP, area postrema; B4, B4 serotonin cells; Bötz, Bötzinger complex; IO, inferior olive; IRt, intermediate reticular nucleus; LC, locus coeruleus; cNTS, caudal nucleus tractus solitarius; Py, pyramidal tract; SubC, subcoeruleus; sp5, spinal trigeminal tract.

After the maximum effect of hypercapnia on firing rate was reached, the perfusate bubbled with $15 \% \mathrm{CO}_{2}$ was changed back to $5 \% \mathrm{CO}_{2}$, and the firing rate also returned to the initial baseline, indicating that the neurons recorded were healthy and the effect of hypercapnia was reversible. For some neurons, the effect of hypercapnia was tested in both aCSF and SNB solution. To assure that we were studying a retrogradely labeled neuron, the pipette solution contained 2\% Lucifer Yellow (LY). For whole-cell configuration, it took at least $30 \mathrm{~min}$ to complete an experiment and this was sufficient for the neuron to be well loaded with LY. For cell-attached configuration, after recording, a rapid negative suction was applied to get into whole-cell mode and the neuron was allowed to load with LY for at least $20 \mathrm{~min}$. At the end of all electrophysiology experiments, slices were fixed in $4 \%$ paraformaldehyde for later confocal imaging to identify rhodamine and LY labeled neurons. All electrophysiology experiments were performed at about $35^{\circ} \mathrm{C}$.

\section{Confocal microscopy}

Fixed slices were washed $3 \times$ for 15 min each in $0.1 \mathrm{M}$ PBS (see Solutions). The washed slice was placed on a glass slide and covered with Vectashield Mounting
Medium designed for fluorescence (Vector Laboratories). The slice was then covered with a cover slip. Fluorescence images were collected with an Olympus FV300 Confocal Microscope (Olympus Corporation) with a $60 \times$ oil objective. Rhodamine bead fluorescence was visualized with excitation light of $568 \mathrm{~nm}$ (from a krypton/argon combination laser) and LY fluorescence was visualized with excitation light of $488 \mathrm{~nm}$ (from a krypton/argon combination laser). Digital images were collected as $Z$ stacks $(0.5 \mu \mathrm{m}$ resolution). The image files were managed with Fluoview software (Olympus Corporation). All confocal images shown are merged $Z$ stack images to show all the rhodamine beads within a soma.

\section{Solutions}

aCSF was composed of (in $\mathrm{mM}$ ): $3 \mathrm{KCl}, 124 \mathrm{NaCl}, 1.3$ $\mathrm{MgSO}_{4}, 26 \mathrm{NaHCO}_{3}, 1.24 \mathrm{NaH}_{2} \mathrm{PO}_{4}, 10$ glucose, and $2.4 \mathrm{CaCl}_{2}$, equilibrated with $95 \% \mathrm{O}_{2} / 5 \% \mathrm{CO}_{2}$ gas, $\mathrm{pH}$ $\sim 7.45$. SNB was the same for aCSF except for (in $\mathrm{mM}$ ): $121 \mathrm{NaCl}, 11.4 \mathrm{MgSO}_{4}$, and $0.2 \mathrm{CaCl}_{2}$, plus carbenoxolone (CAR; $100 \mu \mathrm{M}$ ) to block gap junctions. Hypercapnic solutions were identical to aCSF and SNB solutions except for equilibration with $85 \% \mathrm{O}_{2} / 15 \% \mathrm{CO}_{2}$ gas, $\mathrm{pH} \sim 6.9-7.0$. Paraformaldehyde solutions were 
freshly diluted $1: 1$ from an $8 \%$ paraformaldehyde stock into $100 \mathrm{mM}$ PBS solution, which contained (in $\mathrm{mM}$ ): $137 \mathrm{NaCl}, 2.7 \mathrm{KCl}, 4.3 \mathrm{Na}_{2} \mathrm{HPO}_{4}$, and $1.47 \mathrm{NaH}_{2} \mathrm{PO}_{4}$, $\mathrm{pH}$ 7.4. The whole-cell pipette filling solution contained (in $\mathrm{mM}$ ): $130 \mathrm{~K}$-gluconate, $0.4 \mathrm{EGTA}, 1 \mathrm{MgCl}_{2}, 0.3 \mathrm{GTP}$, 2 ATP, and 10 HEPES, plus $2 \%$ LY. The pipette filling solution $\mathrm{pH}$ was adjusted to $\sim 7.35$ using $\mathrm{KOH}$.

\section{Data analysis and statistics}

For anatomy study, the labeling intensity was determined as follows: $0-1$ neuron identified was assigned the number " 0 ". In the same way, 2-5 neurons were designated as "1"; 6-10 neurons designated as "2"; and more than 10 neurons designated as " 3 "; three animals per group were scored. For the pattern of labeling, all regions with high intensity of labeling (number 2 or 3 ) after injections into the LC or into the cNTS, were assessed to see if these areas have been associated with ventilatory control. Thus, each heavily loaded area was carefully evaluated for the location of the retrograde labeling within the nucleus. From the total amount of labeling within the region, the labeling in the nucleus was subdivided into dorsal, lateral and/or ventral portions, as well as the location rostral-caudal relative to bregma.

The firing rate of rhodamine-loaded LC and CNTS neurons was calculated as the integrated firing rate over a $10-\mathrm{s}$ bin using Clampfit 10.3 software. Thus, the number of action potentials was determined for each 10 -s period and plotted as the number of firings per $10 \mathrm{~s}$ vs. time plots. For each neuron, the average firing rate over a 1-min period was determined either in $5 \% \mathrm{CO}_{2}$ or $15 \% \mathrm{CO}_{2}$, and plotted as firing rate per $\mathrm{s}(\mathrm{Hz})$. All values were expressed as mean \pm SEM, when we had at least three neurons. Significance was set as $P \leqslant 0.05$ and significant differences were determined using paired $T$-tests.

\section{RESULTS}

\section{Anatomy}

Microinjection of rhodamine beads into the $L C$. To assess specificity of retrograde labeling from the LC, the results were divided into four groups (Table 1): (1) LC + : microinjection of rhodamine beads centered into the LC (Fig. 3A); (2) LC Miss: injection site centered ventrally and/or laterally from the LC located in the

Table 1. Numbers of retrogradely labeled neurons in various brainstem regions from rhodamine injections into either the LC or the cNTS

\begin{tabular}{|c|c|c|c|c|c|c|}
\hline & $\mathrm{LC}^{+}$ & LC + Cereb & Peri LC & LC Miss & cNTS $^{+}$ & cNTS Miss \\
\hline \multicolumn{7}{|l|}{ Hypothalamus } \\
\hline Dorsomedial Hypothalamus & 2 & 2 & 2 & 0 & 2 & 0 \\
\hline Lateral hypothalamus & 2 & 2 & 1 & 1 & 2 & 0 \\
\hline Perifornical area & 0 & 1 & 0 & 0 & 0 & 0 \\
\hline Posterior Hypothalamus & 1 & 1 & 2 & 0 & 1 & 0 \\
\hline \multicolumn{7}{|l|}{ (II) MESENCEPHALON } \\
\hline Periaqueductal gray & 3 & 2 & 2 & 0 & 2 & 1 \\
\hline \multicolumn{7}{|l|}{ (III) PONS } \\
\hline Dorsal Raphé & 2 & 1 & 1 & 0 & 1 & 0 \\
\hline LC contralateral or LC & 3 & 2 & 2 & 0 & 2 & 1 \\
\hline Subcoeruleus & 3 & 3 & 1 & 0 & 3 & 0 \\
\hline Parabrachial nuclei & 3 & 2 & 3 & 0 & 0 & 0 \\
\hline Kölliker-Fuse & 3 & 2 & 2 & 0 & 2 & 0 \\
\hline A5 & 2 & 3 & 2 & 0 & 3 & 0 \\
\hline Paragigantocellular nucleus & 2 & 0 & 1 & 0 & 1 & 1 \\
\hline \multicolumn{7}{|l|}{ (IV) MEDULLA } \\
\hline $\mathrm{Amb} / \mathrm{RVL} / \mathrm{CVL}$ & 2 & 2 & 2 & 0 & 2 & 1 \\
\hline Area postrema & 1 & 1 & 1 & 0 & ND & ND \\
\hline Pre-Bötz/Bötz & 2 & 1 & 1 & 0 & 2 & 0 \\
\hline Caudal NTS & 3 & 3 & 3 & 0 & ND & ND \\
\hline Facial & 2 & 1 & 2 & 0 & 1 & 0 \\
\hline Intermediate NTS & 2 & 1 & 2 & 0 & 3 & 1 \\
\hline Inferior olive & 3 & 2 & 1 & 1 & 1 & 0 \\
\hline LRtPC/LRt & 3 & 3 & 1 & 0 & 2 & 0 \\
\hline Medullary raphé & 3 & 3 & 3 & 0 & 1 & 0 \\
\hline Pyramidal tract & 3 & 2 & 2 & 0 & 1 & 2 \\
\hline Retrotrapezoid nucleus & 3 & 3 & 2 & 1 & 2 & 0 \\
\hline Spinal trigeminal tract & 2 & 2 & 2 & 2 & 2 & 2 \\
\hline SpVe/Mve & 1 & 2 & 1 & 0 & 1 & 0 \\
\hline
\end{tabular}

Scoring: "0": 0-1 neuron identified; "1": 2-5 neurons identified. The numbers in black represents a weak labeling pattern. "2": 6-10 neurons identified. The numbers in green represents a median labeling pattern. " 3 ": $>10$ neurons identified. The numbers in red represents a strong labeling pattern. Highlighted areas in yellow represent regions involved in ventilatory control. $N=3$; ND, not determined. 
A Brightfield

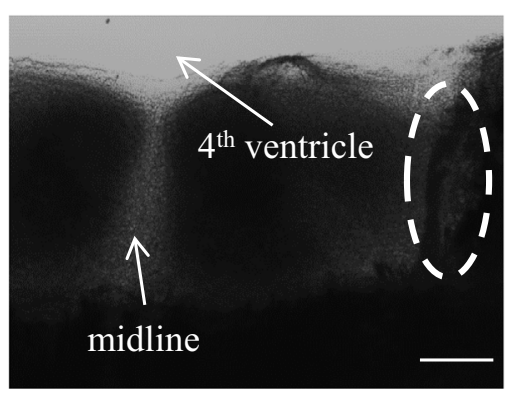

B
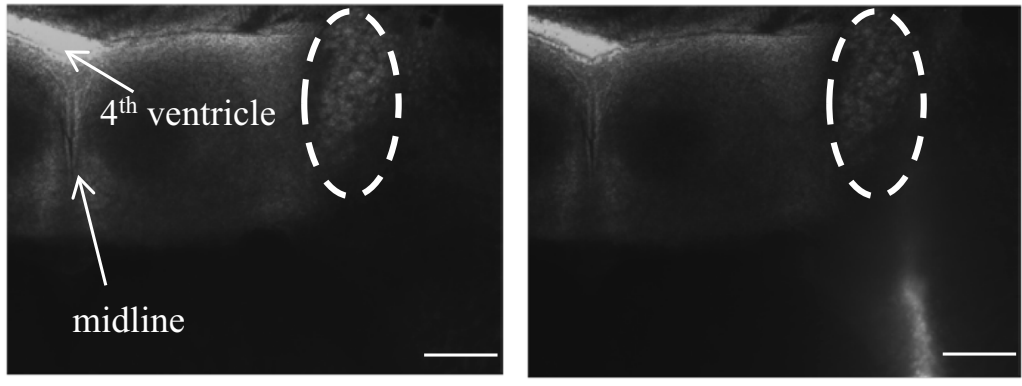

Brightfield and Fluorescent
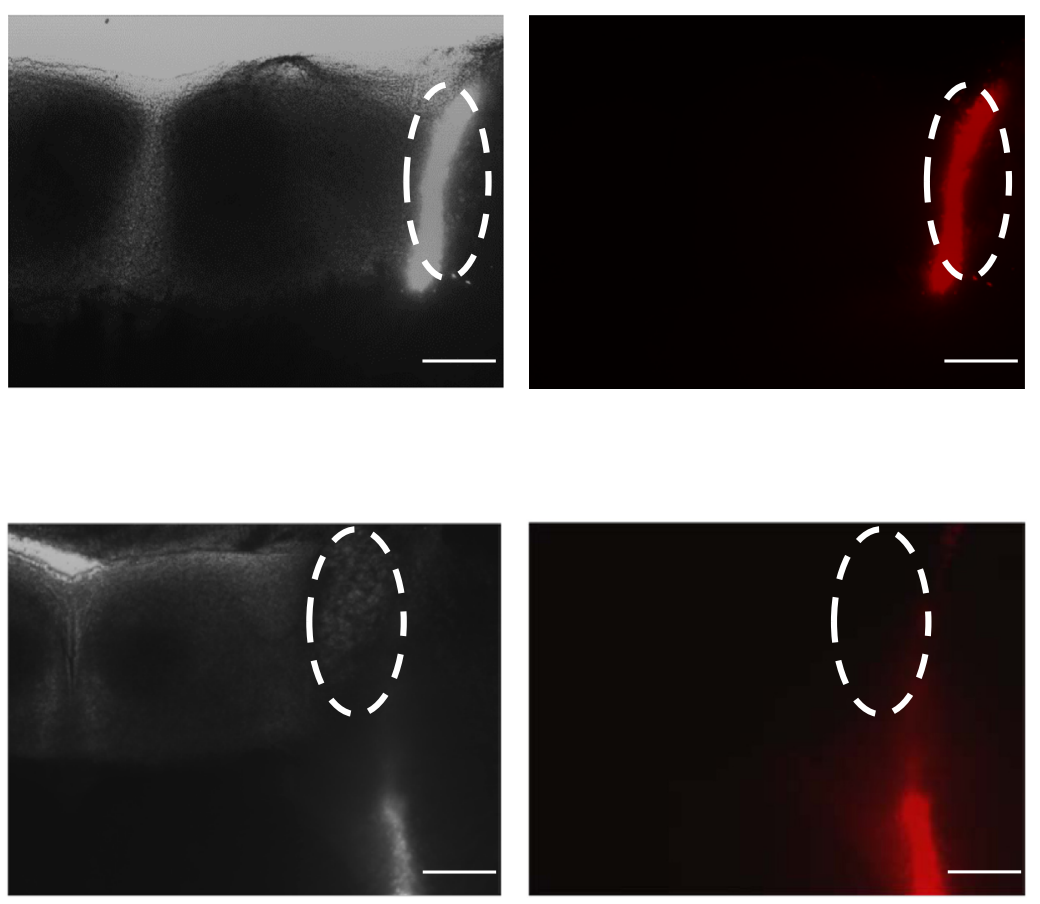

C
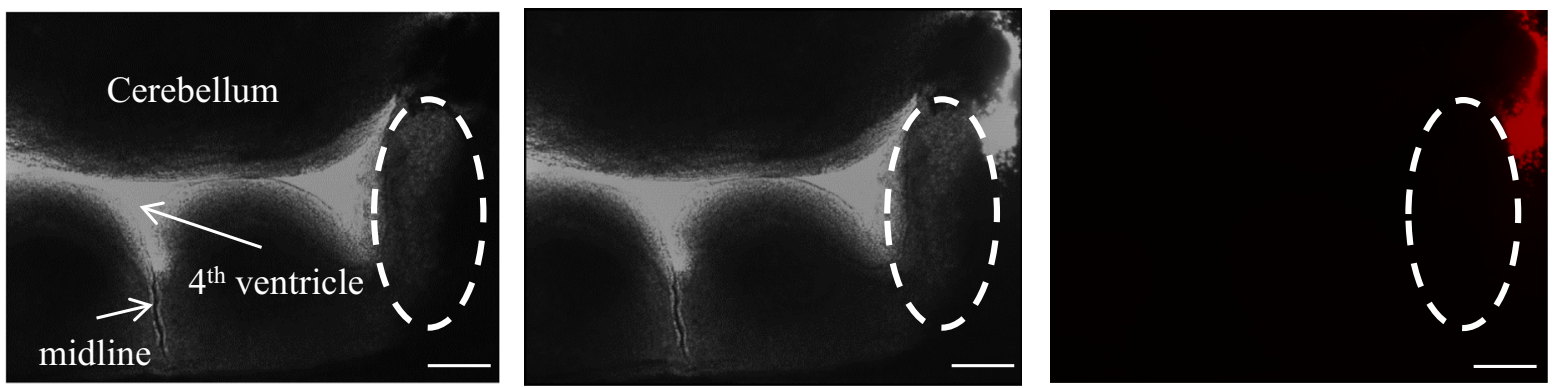

D
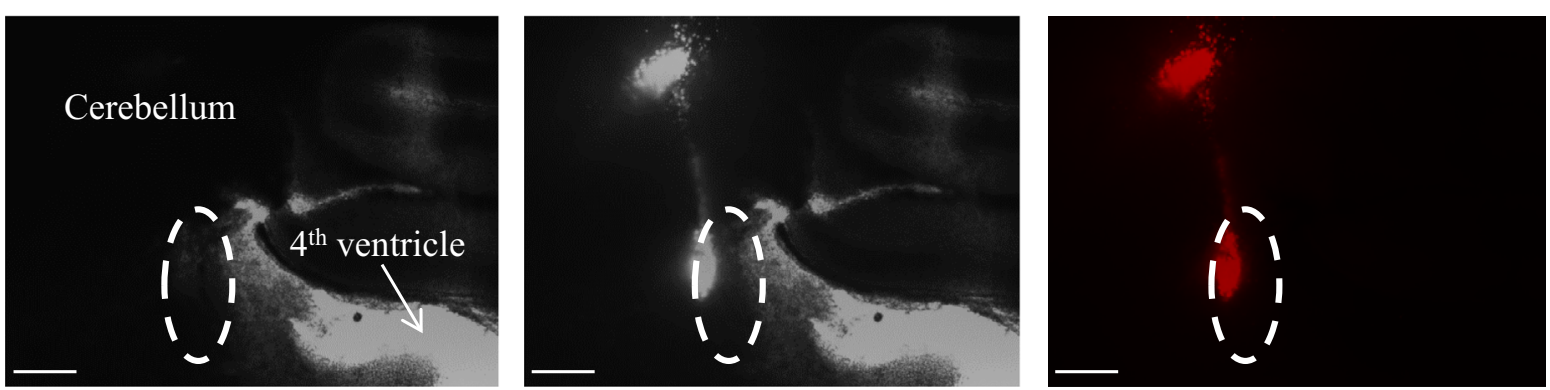

Fig. 3. Microinjection of rhodamine beads in the LC of neonatal rats. The left column shows brightfield images, the middle column shows brightfield and fluorescence imagens and the right column shows fluorescence images of a pontine slice containing the LC. For orientation, the floor of the 4 th ventricle and the midline of the slice are indicated (white arrows) and the location of the LC is outlined by the white dotted ellipse. (A) LC Hit. Note in the fluorescence image that the rhodamine beads (red fluorescence) lie entirely within the LC. (B) LC-Miss. Note in the fluorescent image that the rhodamine beads lie entirely out, ventrally of the LC. (C) Peri-LC. Note in the fluorescent image that the rhodamine beads do not lie within the LC but in an adjacent area abutting the LC , or the peri-LC. (D) LC and Cerebellum Hit. Note in the fluorescent image that the rhodamine beads lie in the LC as well as within the cerebellum. The calibration bar in each image represents $300 \mu \mathrm{m}$. 


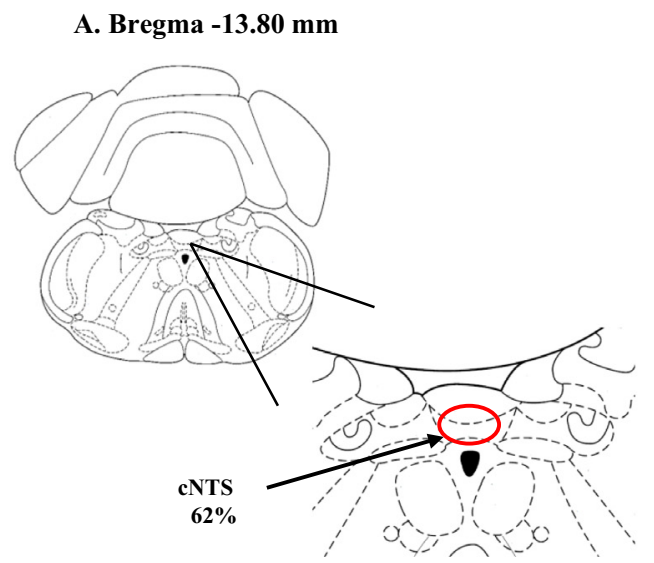

B. Bregma $-7.80 \mathrm{~mm}$

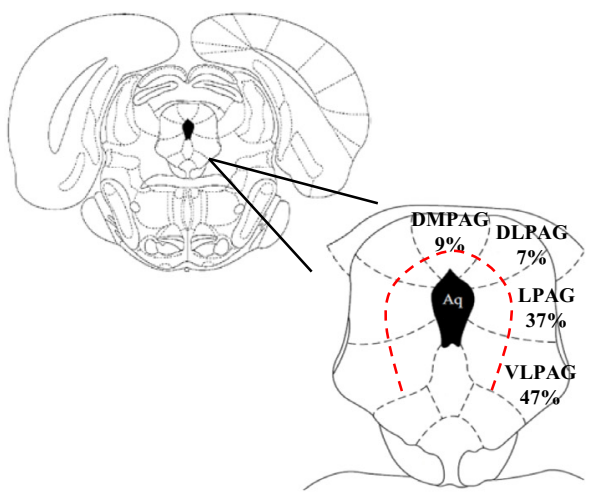

D. Bregma $-9.80 \mathrm{~mm}$

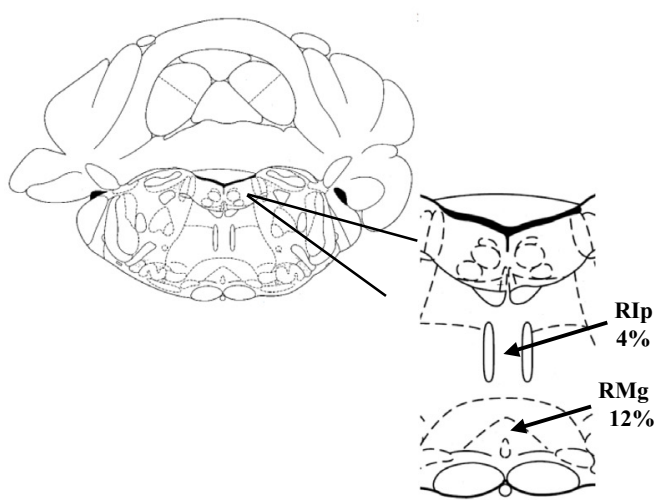

C. Bregma $-12.80 \mathrm{~mm}$

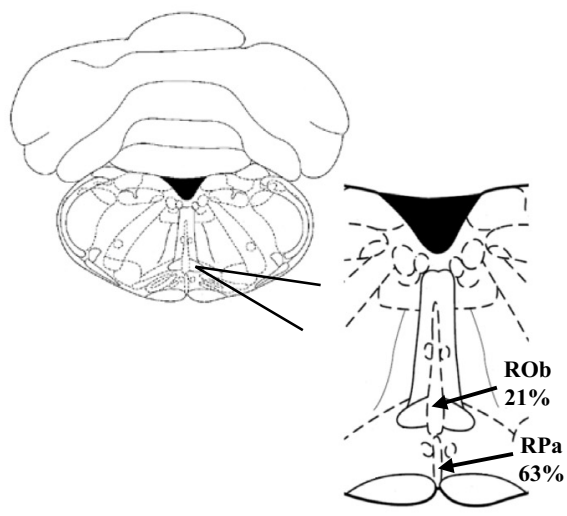

E. Bregma $-10.52 \mathrm{~mm}$

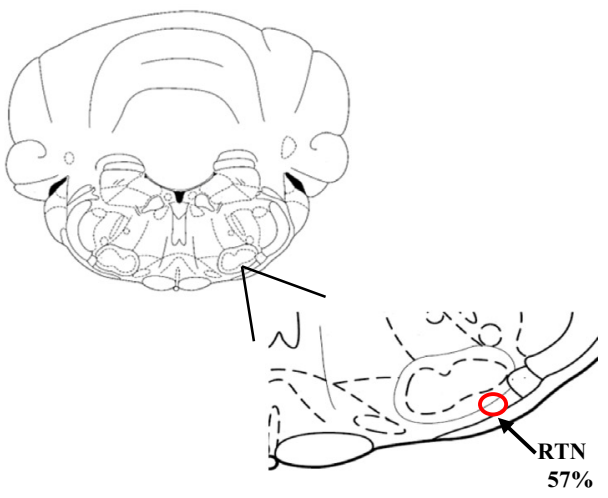

Fig. 4. Schematic drawings of different brain regions showing the pattern of location of retrogradely labeled neurons within certain nuclei from neonatal rats injected with rhodamine beads into LC. The schematic drawings are taken from Paxinos and Watson (1997). (A) cNTS: caudal nucleus tractus solitaries; 162 neurons with rhodamine-loaded soma were counted in 12 slices between Bregma -13.80 and -14.40 mm; of these, $62 \%$ neurons were localized to the dorsolateral, commissural and medial portion of the cNTS (red circle). (B) PAG: periaqueductal gray: 90 neurons with rhodamine-loaded soma were counted in 18 slices between Bregma -7.30 and $-8.80 \mathrm{~mm}$; of these, $9 \%$ were localized to the dorsomedial portion (dmPAG), $7 \%$ to the dorsolateral portion (dIPAG), $37 \%$ to the lateral portion (IPAG) and $47 \%$ to the ventrolateral portion (vIPAG) (area defined by dashed red line). (C) RPa: raphe pallidus nucleus, ROb: raphe obscurus nucleus; 65 neurons with rhodamine-loaded soma were counted in 14 slices between Bregma -9.72 and $-14.16 \mathrm{~mm}$; of these $63 \%$ were in the raphe pallidus nucleus and $21 \%$ of these were in the raphe obscurus nucleus. (D) RMg: raphe magnus nucleus, RIp: raphe interpositus nucleus; 12 neurons with rhodamine-loaded soma were counted in 12 slices between Bregma -9.6 and $-12.12 \mathrm{~mm}$; of these $4 \%$ were localized to the raphe interpositus and $12 \%$ to the raphe magnus. (E) RTN: retrotrapezoid nucleus; 37 neurons with rhodamine-loaded soma were counted in nine slices between Bregma -10.44 and $-10.88 \mathrm{~mm}$; of these $57 \%$ were in the caudal region of the RTN (red circle). (F): Pre-Bötz: Pre-Bötzinger complex; 20 neurons with rhodamine-loaded soma were counted in six slices between Bregma -12.30 and $-12.84 \mathrm{~mm}$; of these $70 \%$ were in the dorsal part of the Pre-Bötz Complex (red circle). (G) Bötz: Bötzinger complex; 27 neurons with rhodamine-loaded soma were counted in six slices between Bregma -11.96 and $-12.48 \mathrm{~mm}$; of these $70 \%$ were in the dorsal part of the Bötz Complex (red circle). (H) IHyp: lateral hypothalamus; 38 neurons with rhodamine-loaded soma were counted in 12 slices between Bregma -1.20 and $-4.56 \mathrm{~mm}$; of these $70 \%$ were in the dorsal part of the Bötz Complex (red circle). (I) cLC: contralateral LC; 19 neurons with rhodamine-loaded soma were counted in 12 slices between Bregma -8.88 and $-10.04 \mathrm{~mm}$; of these $53 \%$ were in the dorsal part of the contralateral LC (red circle). 

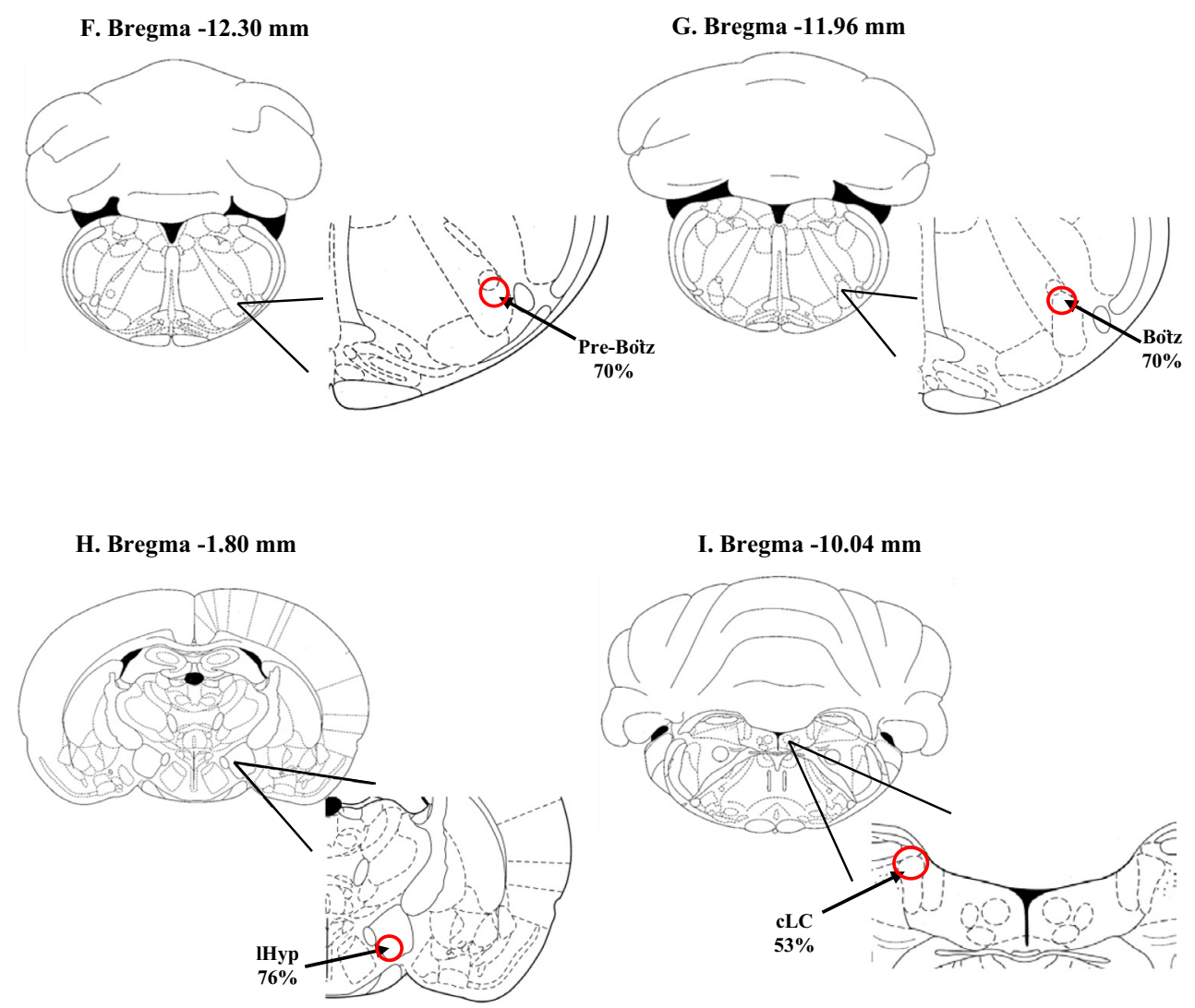

Fig. 4 (continued)

medial vestibular nucleus, magnocellular part (MVeMC), dorsomedial spinal trigeminal nucleus (DMsp5), or peritrigeminal zone/parvicellular reticular nucleus, alpha part (P5/PCRtA) (Fig. 3B); (3) Peri LC: microinjection of rhodamine beads located in the region surrounding the LC (Fig. 3C); and (4) LC + Cereb: microinjection placed within the LC, but with vestiges of rhodamine in the Cereb (Fig. 3D).

$L C+$ group: substantial numbers of rhodaminelabeled cells (See Table 1, "3" in red) were located in a variety of regions thought to be involved in ventilatory control (yellow rows), including the periaqueductal gray (PAG), contralateral LC (cLC), subcoeruleus region (SubC), parabrachial nucleus (PB), Kölliker-Fuse nucleus (KF), cNTS, medullary raphe (MR) and RTN. Moderate labeling (see Table 1, "2" in green) was found in other chemosensitive regions such as the dorsomedial and lateral hypothalamus (dHyp and IHyp respectively), A5 region, $A m b / R V L / C V L$ region (ambiguus nucleus/rostroventrolateral reticular nucleu s/caudoventrolateral reticular nucleus), Pre-Bötzinger and Bötzinger complex (Pre-Bötz/Bötz). We found strong labeling in some non-chemosensitive areas such as inferior olive (IO), lateral reticular nucleus, parvicellular part/lateral reticular nucleus (LRtPC/LRt) and pyramidal tract (Py), and moderate labeling in nonchemosensitive areas such as the Facial Nucleus, Paragigantocellular nucleus, intermediate NTS (iNTS) and spinal trigeminal tract (sp5). Areas with lower intensity of labeling with scattered cells (see Table 1,
"1" or "0" in black) were found only in nonchemosensitive areas (Table 1), including: AP, posterior hypothalamus (pHyp), DR, IO, lateral reticular nucleus, LRtPC/LRt, Py, and spinal vestibular nucleus/medial vestibular nucleus (SpVe/Mve).

$L C+$ Cereb: the pattern of retrograde labeling was very similar to the LC+ group, as expected (Table 1).

Peri LC: retrograde labeling was moderate, with a reduction for some areas compared with the LC+ group, but still present in most regions (Table 1).

LC Miss: there was almost no retrograde labeling in the regions examined (Table 1 ).

These findings suggest that the neuronal projections seen with retrograde labeling are largely specific to the $\mathrm{LC}$ region.

Pattern of retrograde labeling: afferent projections to $L C$. We focused our analysis of retrograde labeling location in nuclei in which moderate (2) to intense (3) labeling were observed after rhodamine bead injection into the LC. Only animals belonging to the LC + group and the LC Miss group (control) were considered.

Among the chemosensitive areas with such retrograde labeling is the cNTS. In the cNTS, the dorsolateral, commissural and medial portion of the nucleus was the region with majority neurons loaded with rhodamine $(62 \%)$, while $38 \%$ of labeled neurons were observed in the interstitial, intermediate, ventral and ventrolateral regions (Fig. 4A). 
The second region with higher intensity of retrograde labeling was the PAG. Most of the labeled neurons were present in the ventrolateral and lateral PAG, about $47 \%$ and $37 \%$, respectively (Fig. 4B). As for the other subdivisions, in the dorsomedial and dorsolateral PAG less than $10 \%$ of labeling was observed. Fig. 4B also shows that approximately $64 \%$ of all PAG labeling was found near the aqueduct to the medial region.

The MR was the third region with a large number of retrogadely labeled neurons. Of the loaded neurons in this region, $63 \%$ were in the raphe pallidus, $21 \%$ were in the raphe obscurus, $12 \%$ in the raphe magnus and only $4 \%$ were in the raphe interpositus nucleus (RIp) (Fig. 4C, D). In the RTN, $57 \%$ of the labeled neurons occurred in the caudal region of the nucleus, specifically in the lateral portion (Fig. 4E).

Other regions that have moderate labeling patterns were the IHyp, Pre-Bötz and Bötz region. In the PreBötz and Bötz region, most of the labeled neurons were located in the dorsal portion, as shown in Fig. 4F, G. For the IHyp, $76 \%$ of the labeled neurons were located in the ventral portion (Fig. $4 \mathrm{H}$ ). However, this pattern was only observed for more rostral coordinates, since for the caudal region of $\mathrm{IHyp}$ a more homogeneous distribution occurred. Also in the CLC, $53 \%$ of the labeled cells were found in the dorsal portion of the nucleus, followed by the ventral and middle portion, $26 \%$ and $21 \%$ respectively (Fig. 4I).

It was observed that other nuclei, such as dHyp, SubC, PB, KF, A5 region and $A M B / R V L / C V L$ had scattered projections to LC but no particular loading pattern was noted. Also, for the LC Miss group none of the patterns observed in the LC+ group were found.

Microinjection of rhodamine beads into the cNTS. The results were divided into two groups: (1) cNTS + : microinjection of rhodamine beads centered into the cNTS (Fig. 5A); and (2) cNTS Miss: injection site located out of the cNTS, placed in the cuneate nucleus and gracile nucleus (Fig. 5B).

cNTS +: chemosensitive regions containing rhodamine fluorescence with high density (Table 1, "3" in red) were found only in the SubC and A5 regions. We found strong labeling also in the non-chemosensitive iNTS. Moderate labeling (Table 1, "2" in green) was observed in chemosensitive areas such as the dHyp and IHyp, PAG, LC, KF, Amb/RVL/CVL, Pre-Bötz/Bötz and RTN. Moderate labeling was observed also in two non-chemosensitive areas, the LRtPC/LRt and the sp5. Chemosensitive areas with lower intensity of labeling with scattered or individual cells (Table 1, " 0 " or " 1 " in black) were found in PB and MR. The neurons
A

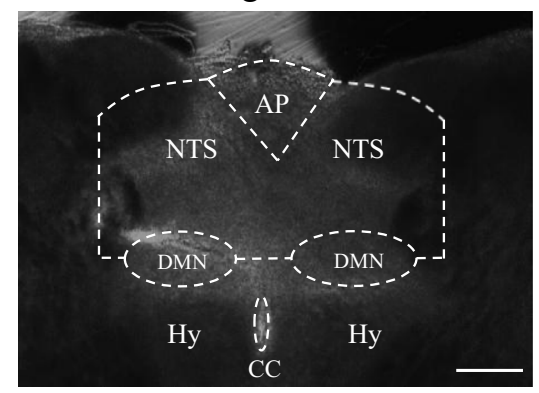

Brightfield

B

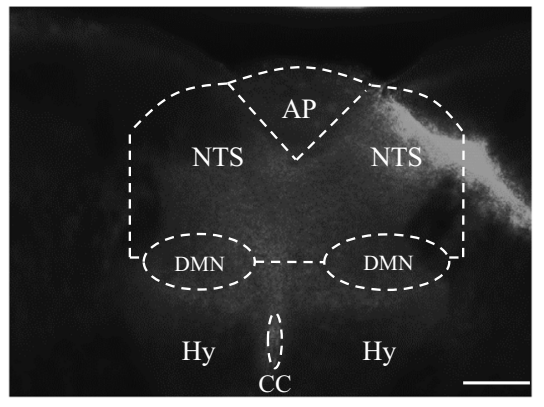

Brightfield and Fluorescent
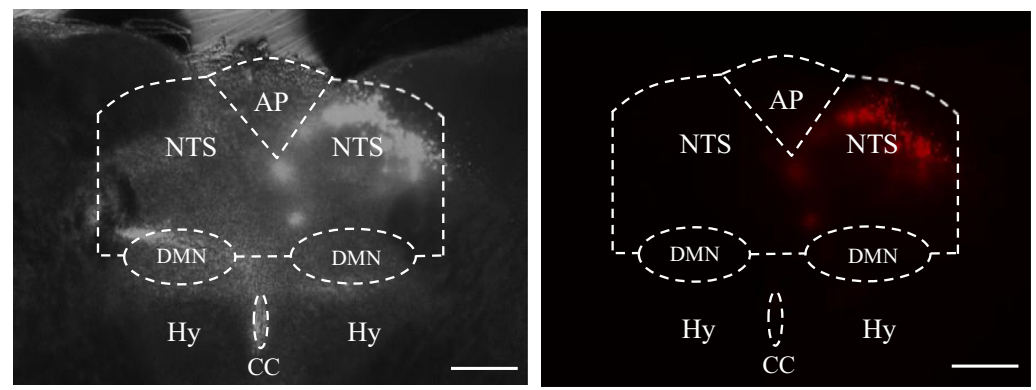

Fluorescent

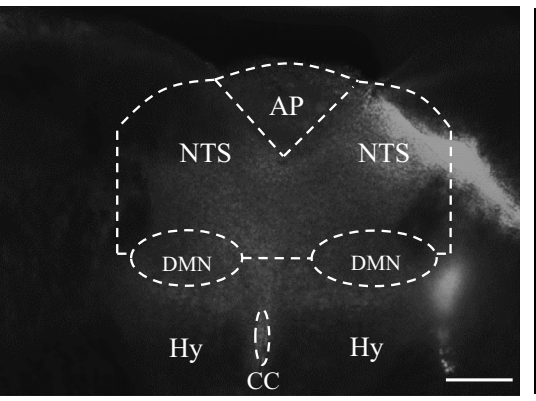

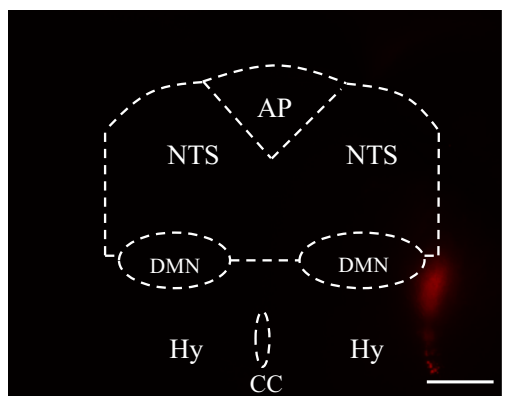

Fig. 5. Microinjection of rhodamine beads into the cNTS of neonatal rats. The left column shows brightfield images, the middle column shows brightfield and fluorescence imagens and the right column shows fluorescence images of a medullary slice containing the cNTS. For orientation, the general area of the hypoglossal ( $\mathrm{Hy}$ ) nucleus is marked, and the central canal (CC), the area postrema (AP), the dorsal motor nucleus of the vagus (DMN) and the NTS are delineated by white dotted lines. (A) NTS Hit. Note in the fluorescence image that the rhodamine beads (red fluorescence) lie entirely within the NTS. (B) NTS Miss. Note in the fluorescence image that the rhodamine beads lie entirely outside of the NTS. The calibration bar in each image represents $300 \mu \mathrm{m}$. 


\section{A. Bregma $-10.80,10.30$ and $9.80 \mathrm{~mm}$}

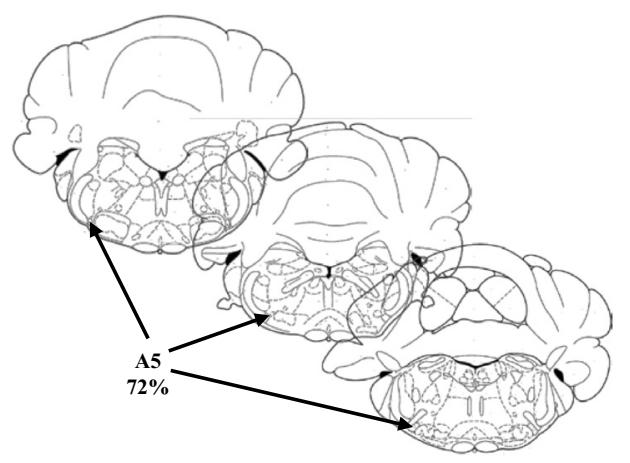

B. Bregma $-9.68 \mathrm{~mm}$

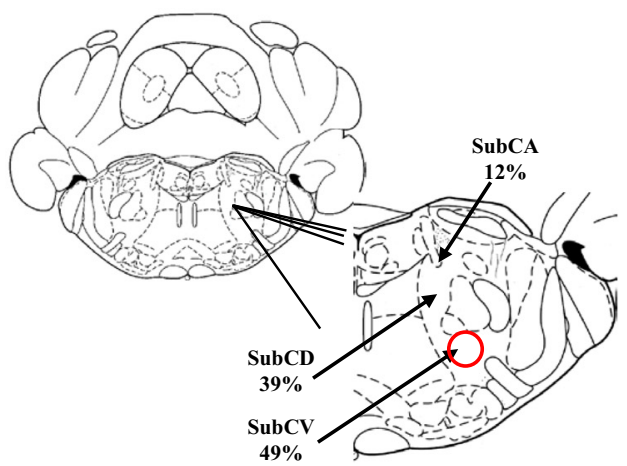

D. Bregma $-7.80 \mathrm{~mm}$

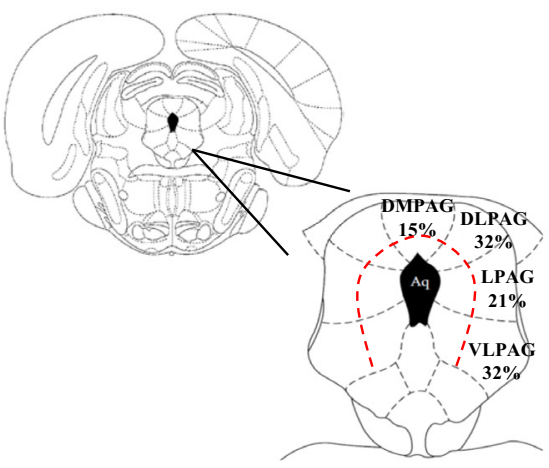

C. Bregma -10.04 mm

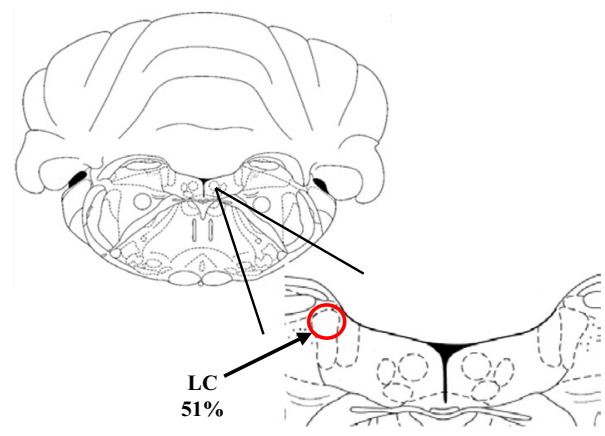

E. Bregma - $-10.52 \mathrm{~mm}$

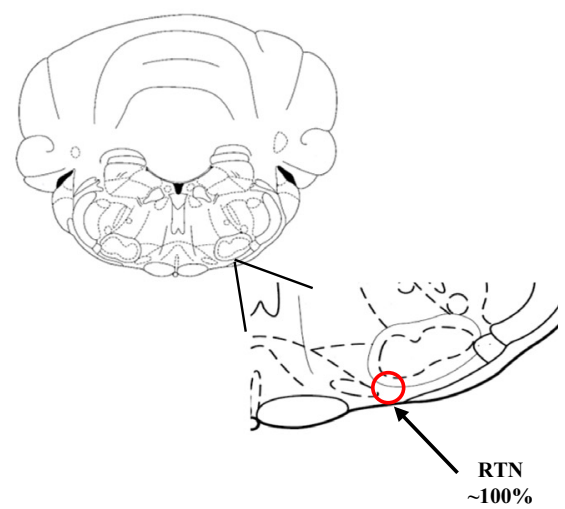

Fig. 6. Schematic drawings of different brain regions showing the pattern of location of retrogradely labeled neurons within the nuclei from neonatal rats injected with rhodamine beads into cNTS. The schematic drawings are taken from Paxinos and Watson (1997). (A) A5 region; 106 neurons with rhodamine-loaded soma were counted in 24 slices between Bregma -9.00 and $-11.28 \mathrm{~mm}$; of these, $72 \%$ were localized to the caudal portion of the A5 region. (B) SubC: subcoeruleus nucleus alpha portion (SubCA), dorsal portion (SubCD) and ventral portion (SubCV); 103 neurons with rhodamineloaded soma were counted in 12 slices between Bregma -8.88 and $-9.96 \mathrm{~mm}$; of these, $12 \%$ were localized to the SubCA region, $39 \%$ to the SubCD region, and $49 \%$ to the SubCV region (red circle). (C) LC: locus coeruleus; 58 neurons with rhodamine-loaded soma were counted in 12 slices between Bregma -8.80 and $-10.04 \mathrm{~mm}$; of these, $51 \%$ were localized to the dorsal region of the LC (red circle). (D) PAG: periaqueductal gray dorsomedial portion (dmPAG), dorsolateral portion (dIPAG), lateral portion (IPAG) and ventrolateral portion (vIPAG); 48 neurons with rhodamine-loaded soma were counted in 18 slices between Bregma -7.30 and $-8.80 \mathrm{~mm}$; of these, $15 \%$ were localized to the dmPAG region, $32 \%$ in the dIPAG region, $21 \%$ in the IPAG region and $32 \%$ in the VIPAG region (all areas defined by the dashed red line). (E) RTN: retrotrapezoid nucleus; 30 neurons with rhodamineloaded soma were counted in 9 slices between Bregma -10.44 and $-10.80 \mathrm{~mm}$; of these, $100 \%$ were localized to the caudal region and central area of the RTN (red circle). (F) KF: Kölliker-Fuse; 41 neurons with rhodamine-loaded soma were counted in six slices between Bregma -8.72 and $-8.80 \mathrm{~mm}$; of these, $83 \%$ were localized to the ventral portion of the KF (red circle). (G) Pre-Bötz: Pre-Bötzinger complex; 64 neurons with rhodamine-loaded soma were counted in six slices between Bregma -12.30 and $-12.84 \mathrm{~mm}$; of these, $72 \%$ were localized to the ventral portion of the Pre-Bötz (red circle). (H) Bötz: Bötzinger complex; 50 neurons with rhodamine-loaded soma were counted in six slices between Bregma -11.96 and $-12.48 \mathrm{~mm}$; of these, $72 \%$ were localized to the ventral portion of the Bötz (red circle). (I) IHyp: lateral hypothalamus. Sixty-three neurons with rhodamine-loaded soma were counted in 18 slices between Bregma -1.2 and $-4.56 \mathrm{~mm}$; of these, $77 \%$ were localized to the ventral portion of the IHyp (red circle). 
F. Bregma $-8.72 \mathrm{~mm}$

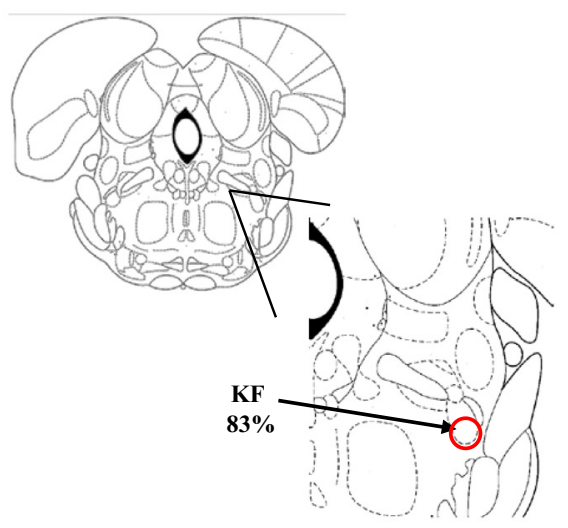

G. Bregma $-12.30 \mathrm{~mm}$

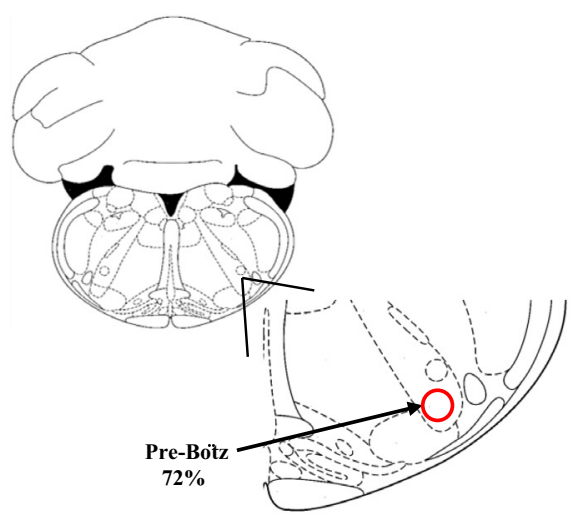

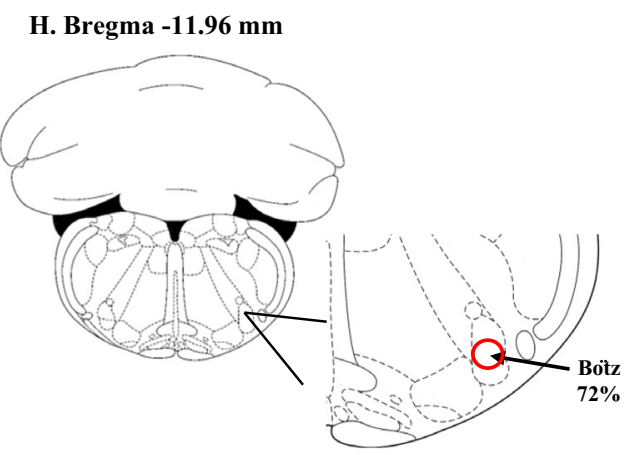

I. Bregma $-1.80 \mathrm{~mm}$

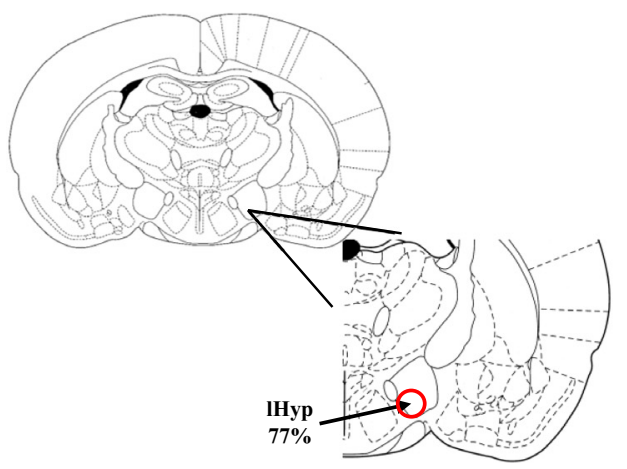

Fig. 6 (continued)

projecting to the NTS from non-chemosensitive areas were from the perifornical area (PA), pHyp, DR, Facial nucleus, IO, Py and SpVe/Mve. The neurons projecting to the NTS from non-chemosensitive areas were the same that projected to the LC.

cNTS Miss: retrograde labeling was nearly absent in cNTS miss when compared to when injections were in cNTS +.

Pattern of retrograde labeling: afferent projections to cNTS. The A5 region and the SubC region both had a similar large number of labeled neurons. For the A5 region, most labeled neurons $(72 \%)$ occurred in the caudal portion (Fig. 6A). For the SubC region, most labeled cells were observed in the ventral part (49\%), followed by the dorsal and alpha part, $39 \%$ and $12 \%$, respectively (Fig. 6B).

With respect to the $\mathrm{LC}$, about $51 \%$ of all labeled neurons occurred in the dorsal portion (Fig. 6C). In addition, $32 \%$ of labeled neurons were seen in the ventral portion followed by the medial portion $(17 \%)$ of the LC.

We also studied the labeling pattern in the PAG, RTN, $K F$, Pre-Bötz and Bötz region, IHyp and dHyp. For the PAG, most of the loaded cells were present in the ventrolateral and dorsolateral PAG subdivision, with
$32 \%$ for each one, following by $21 \%$ in the lateral and $15 \%$ in the dorsal medial PAG subdivision (Fig. 6D). Approximately $75 \%$ of all PAG labeling was found near the aqueduct to the medial region (Fig. 6D). In the RTN nucleus, all labeled neurons were located in the caudal region of the nucleus, specifically in the central area of this nucleus, as highlighted in Fig. 6E. For the KF, 83\% of the retrogradely labeled neurons were found in the ventral portion of the nucleus and the remaining labeled neurons were widespread in the dorsal and medial portion (Fig. 6F). A similar pattern of labeling (ventral prevalence) was present in the Pre-Bötz and Bötz region, since $72 \%$ of the rhodamine-labeled cells were found in this portion of these nuclei (Fig. 6G, H). Finally, the IHyp was the nucleus of the hypothalamic region with the most expressive labeling, wherein $77 \%$ of all cells occurred in the ventral portion, followed by the dHyp (Fig. 6I).

As to $A m b / R V L / C V L$, labeled neurons were observed dispersed throughout the whole nucleus.

For the cNTS Miss group none of these patterns noted in the cNTS + group were found.

Retrograde labeling and LY. Confocal microscopy was performed on selected slices from various regions of the brainstem to confirm that apparent retrograde 

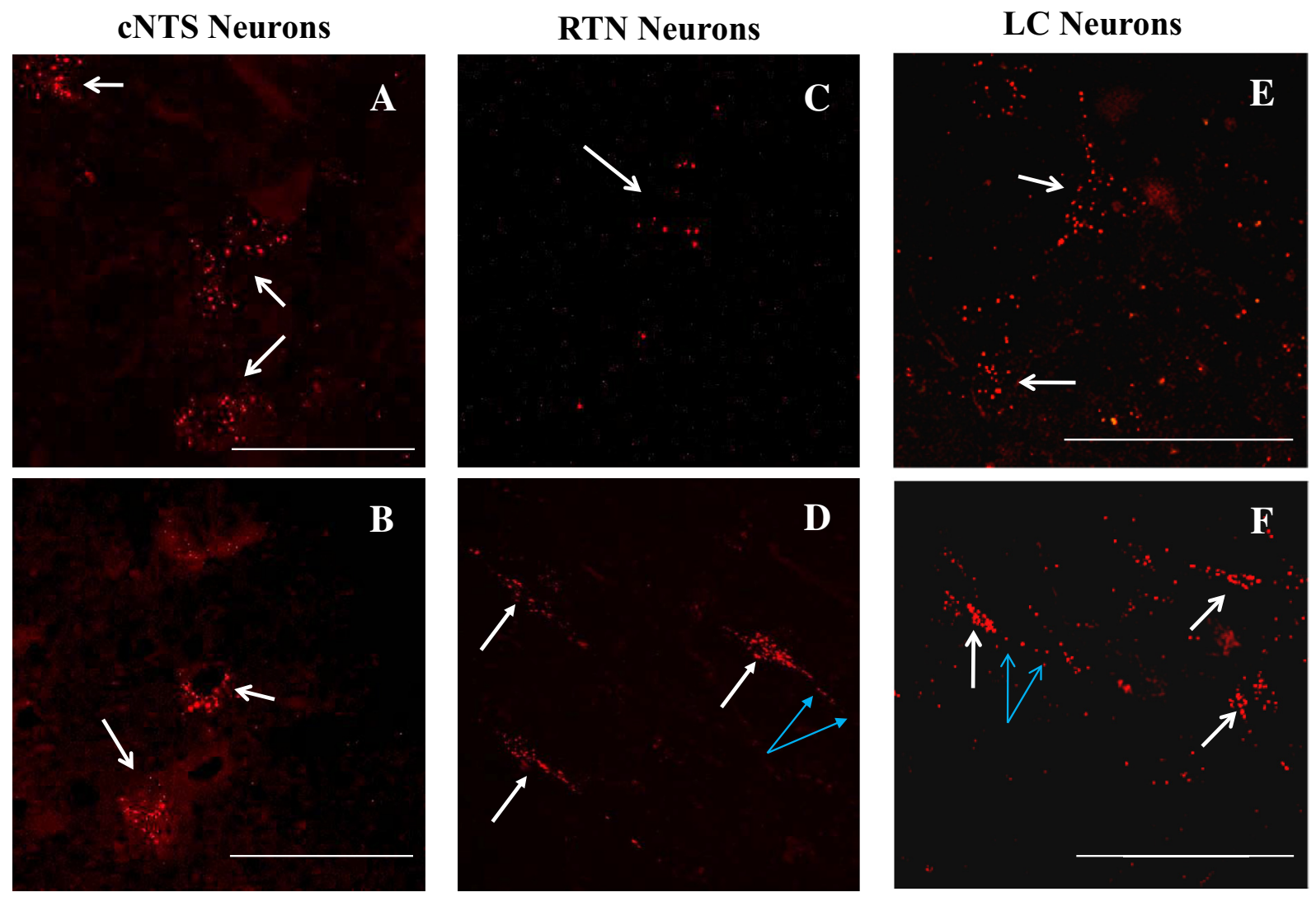

PAG Neurons

Fig. 7. Confocal images of retrogradely labeled neurons after injection of rhodamine stack images. (A, B) Neuronal cell bodies from the cNTS loaded with rhodamine beads (red fluorescence) (white arrows) indicating that neurons from the cNTS have afferent projections to the LC. Note multiple rhodamine beads in each soma indicating heavy retrograde labeling. (C) A neuronal cell body from the area of the RTN loaded with rhodamine beads (red fluorescence) (white arrow). Note there are several rhodamine beads in the soma. (D) Neuronal cell bodies from the PAG loaded with rhodamine beads (red fluorescence) (white arrows) indicating that neurons from the PAG have afferent projections to the LC. Note multiple rhodamine beads in each soma. Also note the presence of rhodamine beads in a neuronal process (blue arrows). (E, F) Neuronal cell bodies from the LC loaded with rhodamine beads (red fluorescence) (white arrows) indicating that neurons from the LC have afferent projections to the cNTS. Note multiple rhodamine beads in each soma indicating heavy retrograde labeling. Also note the presence of rhodamine beads in neuronal processes (blue arrows in F). cNTS, caudal nucleus tractus solitarius; LC, locus coeruleus; PAG, periaqueductal gray; RTN, retrotrapezoid nucleus. The calibration bar in each image represents $50 \mu \mathrm{m}$.

labeling was due to rhodamine bead-labeled cell bodies. Indeed, these regions revealed neuronal cell bodies that contained rhodamine beads (red fluorescence) in the cytoplasm surrounding the nucleus, as well as in the axonal processes of some neurons (Fig. 7), as expected for a dye that loads retrogradely. After injection of rhodamine beads into the LC, several of these redlabeled neurons were visible in the cNTS (Fig. 7A, B; white arrows). Rhodamine-labeled neurons were also seen in the region of the RTN (Fig. 7C, white arrow) and in the PAG, where both loaded soma (white arrows) and axonal processes (blue arrows) were seen (Fig. 7D). Similarly, when rhodamine beads were injected into the cNTS dye-loaded LC neuronal cell bodies (Fig. 7E, F; white arrows) and axonal processes (Fig. $7 \mathrm{~F}$, blue arrows) were seen

These rhodamine-labeled cell bodies were also visible under epifluorescence microscopy during the electrophysiological experiments and were targeted for patch-clamp study. To ensure that the retrogradely labeled neuron was the same that was studied in the electrophysiology protocol, the patched neuron was loaded with LY, added into the patch pipette solution. After the electrophysiological study, the slice was verified in the confocal microscopy to confirm if the neuron studied was positive for both rhodamine and LY labeling. Examples of such neurons from the cNTS and the LC are shown in Fig. 8. The top panel shows neurons from the CNTS that were retrogradely labeled with rhodamine beads (left side; white arrow) that had been injected into the LC, and the same neuron loaded with LY (right side; green fluorescence, white arrow) (Fig. 8A). The neuron indicated by the white arrow is a retrogradely labeled cNTS neuron that was studied electrophysiogically and afterward, loaded with LY (green fluorescence). Another neuron is also visible in the figure (right panel; blue arrow) which represents a neuron with retrograde labeling but without the LY fluorescence, which means that this neuron was not patched and studied for electrophysiological properties. 
A

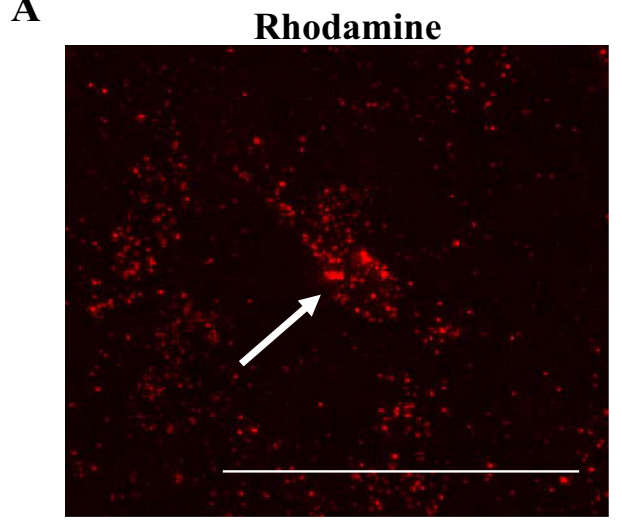

\section{cNTS NEURON}

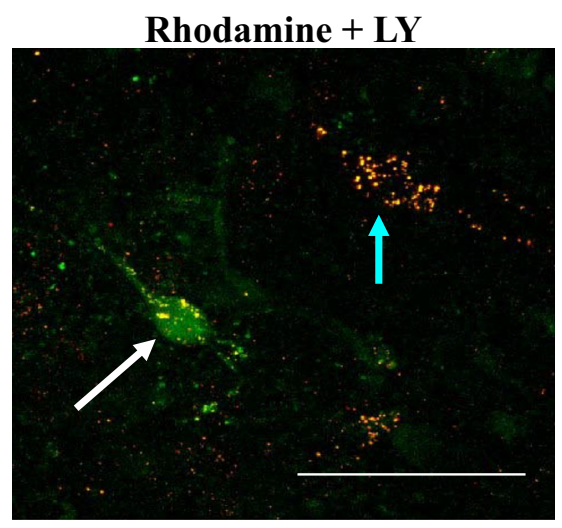

B

\section{LC NEURON}
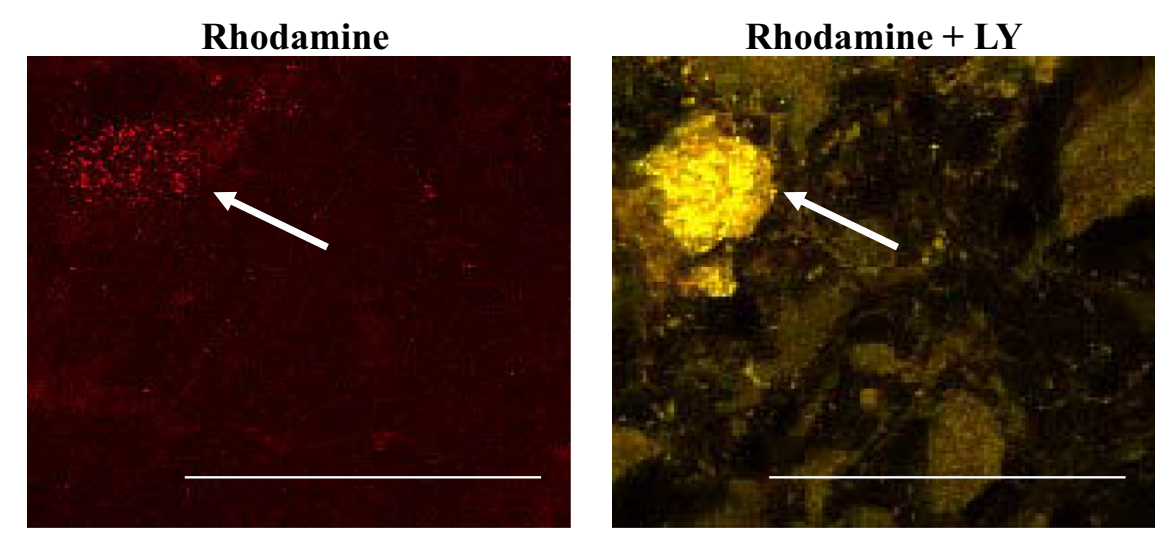

Fig. 8. (A) Neurons from the cNTS that have been retrogradely labeled with rhodamine beads injected into the LC. In the left panel is indicated a cNTS neuronal cell body with numerous rhodamine beads (white arrow). This neuron was patched and studied electrophysiologically and then loaded with Lucifer Yellow (LY). In the right panel the same neuron seen in the left panel shows extensive loading with LY (green fluorescence) with several yellow dots that indicate the overlap of the rhodamine beads seen in the left panel with LY (white arrow). Note a separate neuron that is loaded with rhodamine beads but not with LY (blue arrow). (B) In the left panel is a neuron from the LC that has been retrogradely labeled with rhodamine beads (white arrow) injected into the cNTS. This neuron was studied electrophysiologically and then loaded with LY, but at the end of the recording, the neuron was damaged (white arrow). It is clear that the LY-loaded neuron is in the same location as the rhodamine-labeled neuron in the left panel. All images shown are merged $Z$ stack images. The calibration bar in each image represents $50 \mu \mathrm{m}$.

In the bottom panel, a retrogradely labeled LC neuron is shown (left side; red fluorescence, white arrow) that was labeled with rhodamine beads that had been injected into the cNTS (Fig. 8B). This same neuron was patched, studied electrophysiologically and then injected with LY (right side; yellow fluorescence, white arrow) (Fig. 8B).

\section{Electrophysiology}

Fig. 9A shows that hypercapnic acidosis induced a striking increase in the firing rate of rhodamine-labeled cNTS neurons. This effect was also maintained in SNB solution (Fig. 9B), indicating that this chemosensitive response of firing rate in rhodamine-labeled cNTS neurons is intrinsic and not dependent on synaptic transmission. In four of five patched rhodamine-labeled cNTS neurons, hypercapnic acidosis in SNB solution induced depolarization and a significant increase in firing rate $\left(0.58 \pm 0.3 \mathrm{~Hz}\right.$ in $5 \% \mathrm{CO}_{2}$ vs. $1.26 \pm 0.5 \mathrm{~Hz}$ in $15 \% \mathrm{CO}_{2}, n=4, p=0.04$, Fig. $\left.9 \mathrm{C}\right)$. In one neuron, exposure to hypercapnia caused a hyperpolarization and reduction in firing rate $\left(0.73 \mathrm{~Hz}\right.$ in $5 \% \mathrm{CO}_{2}$ vs. $0.27 \mathrm{~Hz}$ in $15 \% \mathrm{CO}_{2}$, Fig. 9D). This result suggests that the majority of cNTS neurons with projections to the LC appear to be intrinsically chemosensitive.

Fig. 10A shows that hypercapnic acidosis led to an apparent decrease in firing rate of rhodamine-labeled LC neurons. This effect was also maintained in SNB solution (Fig. 10B), suggesting that this rhodaminelabeled LC neuron is intrinsically $\mathrm{CO}_{2}$ chemosensitive. In two of the three neurons, the addition of hypercapnia resulted in a reversible decrease in firing rate $(1.67 \mathrm{~Hz}$ in $5 \% \mathrm{CO}_{2}$ vs. $0.94 \mathrm{~Hz}$ in $15 \% \mathrm{CO}_{2} ; 0.19 \mathrm{~Hz}$ in $5 \% \mathrm{CO}_{2}$ vs. $0.01 \mathrm{~Hz}$ in $15 \% \mathrm{CO}_{2}$, Fig. $10 \mathrm{C}$ ). In the third, firing rate was unchanged or slightly decreased by hypercapnia $\left(0.4 \mathrm{~Hz}\right.$ in $5 \% \mathrm{CO}_{2}$ vs. $0.33 \mathrm{~Hz}$ in $15 \%$ $\mathrm{CO}_{2}$, Fig. 10C). Fig. 10D demonstrates that the firing rate dropped from $1.23 \mathrm{~Hz}$ to $0.014 \mathrm{~Hz}$ by hypercapnia in the same neuron shown in Fig. 10B. These results suggest that the LC neurons with projections to the cNTS may be inhibited by hypercapnia. 


\section{Rhodamine-loaded cNTS neuron}
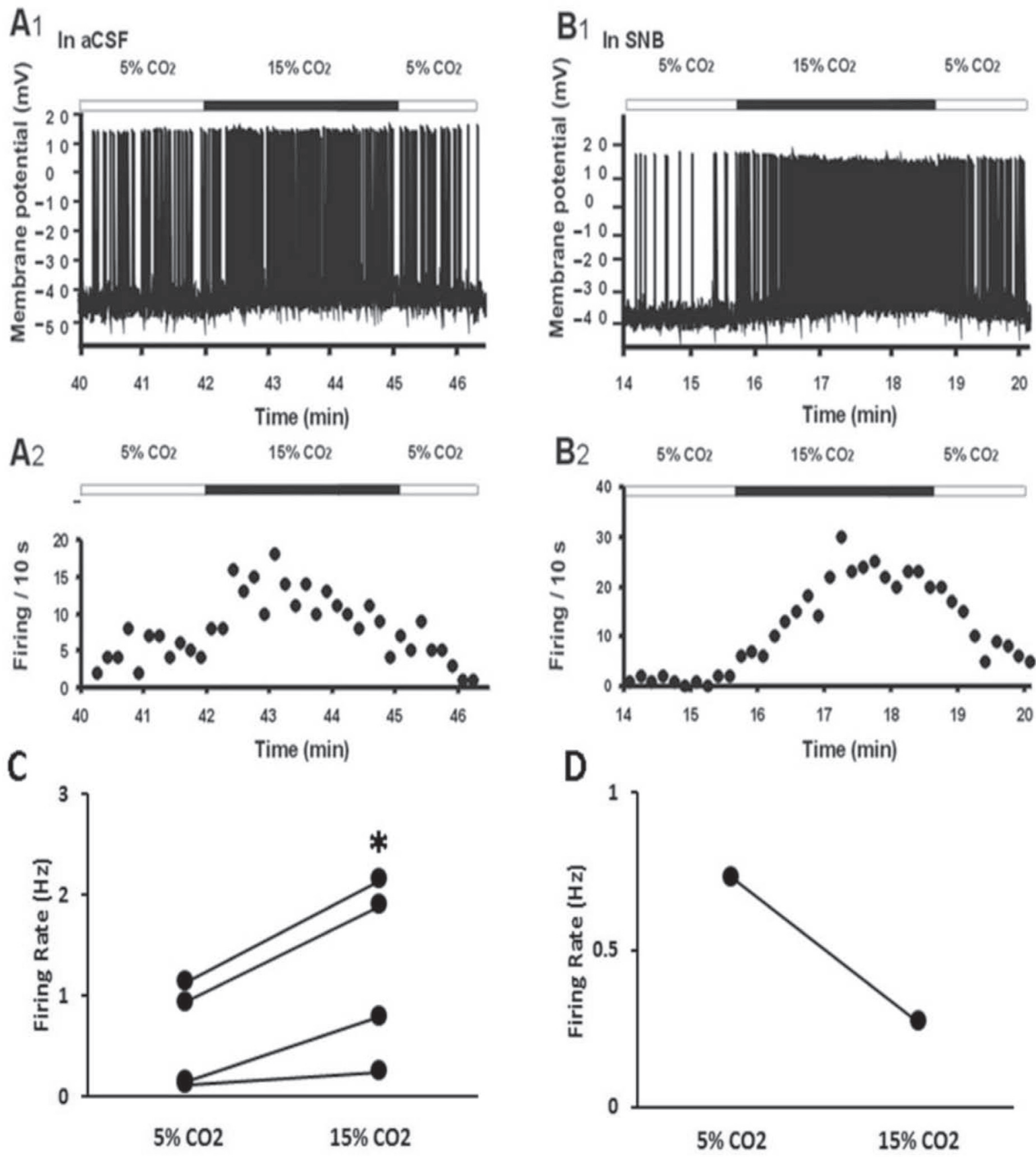

Fig. 9. (A) The firing response to hypercapnia (whole-cell patch in current-clamp mode) of a neuron from the cNTS that contains rhodamine beads retrogradely labeled from the LC, the site of rhodamine bead injection. (A1) The firing rate response to hypercapnic acidotic solution $\left(15 \% \mathrm{CO}_{2}\right)$ in the presence of aCSF. (A2) The integrated firing rate (action potentials per $10 \mathrm{~s})$ in normocapnic $\left(5 \% \mathrm{CO}_{2}\right)$ and hypercapnic $\left(15 \% \mathrm{CO}_{2}\right)$ solutions. (B1) The firing rate response of the same neuron as in $A$ to hypercapnic acidotic solution $\left(15 \% \mathrm{CO}_{2}\right)$ in the presence of synaptic block medium (SNB). (B2) The integrated firing rate of the same neuron as in $\mathrm{A}$ in normocapnic $\left(5 \% \mathrm{CO}_{2}\right)$ and hypercapnic $\left(15 \% \mathrm{CO}_{2}\right) \mathrm{SNB}$ solution. Note that hypercapnia reversibly increased the firing rate of this cNTS neuron that projects to the LC even in the presence of SNB solution. (C) Summary of the effect of hypercapnia in SNB solution on firing rate ( $\mathrm{Hz}$ : action potentials per s) in four rhodamine-labeled cNTS neurons. (D) Hypercapnia caused reduction in firing rate in one rhodamine-labeled cNTS neuron. 


\section{Rhodamine-loaded LC neuron}
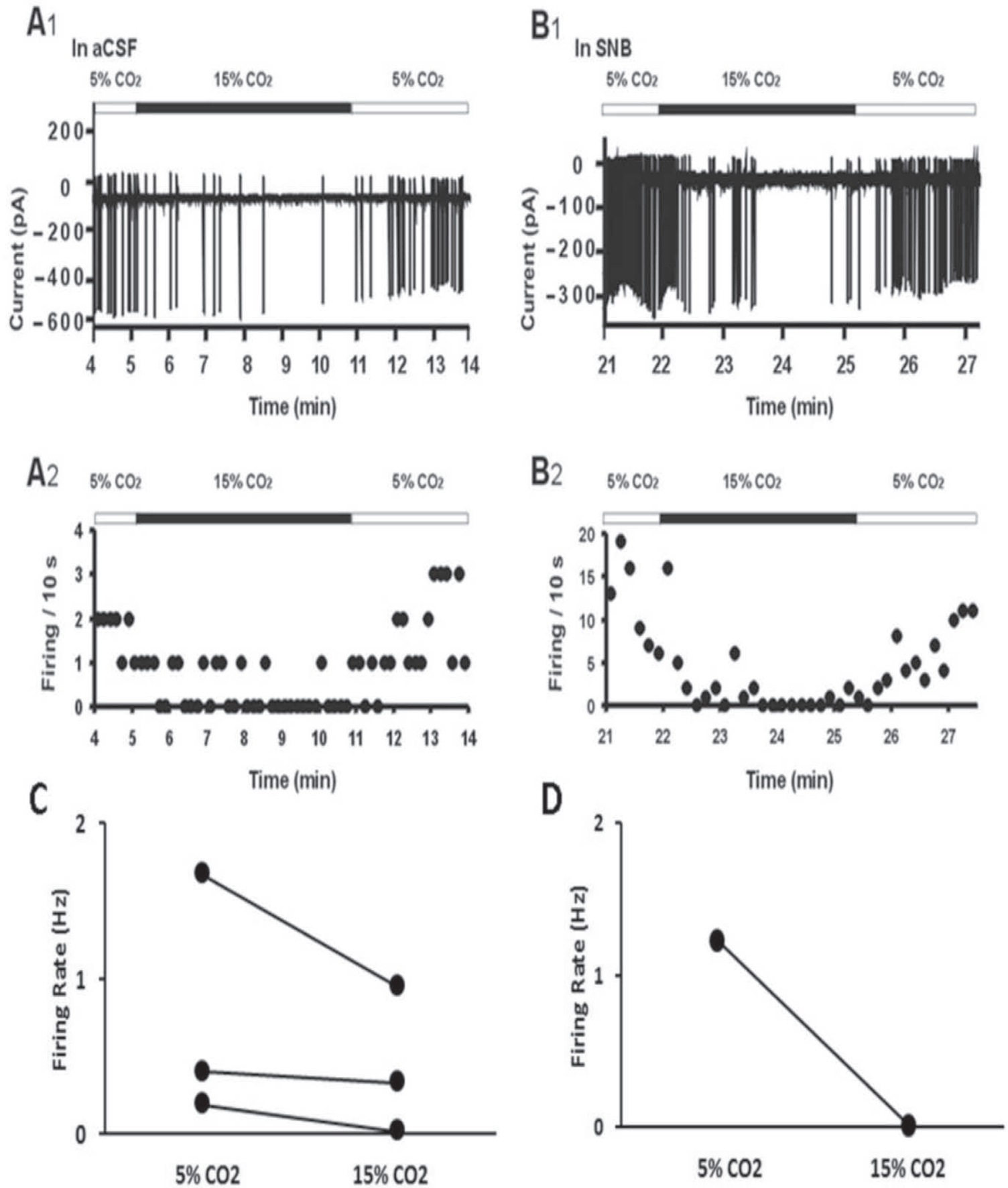

Fig. 10. (A) The firing response to hypercapnia (loose-cell patch in current-clamp mode) of a neuron from the LC that contains rhodamine beads retrogradely labeled from the $\mathrm{CNTS}$, the site of rhodamine bead injection. (A1) The firing rate response to hypercapnic acidotic solution $\left(15 \% \mathrm{CO}_{2}\right)$ in the presence of aCSF. (A2) The integrated firing rate (action potentials per $10 \mathrm{~s})$ in normocapnic $\left(5 \% \mathrm{CO}_{2}\right)$ and hypercapnic $\left(15 \% \mathrm{CO}_{2}\right)$ solutions. (B1) The firing rate response of the same neuron as in A to hypercapnic acidotic solution $\left(15 \% \mathrm{CO}_{2}\right)$ in the presence of synaptic block medium (SNB). (B2) The integrated firing rate of the same neuron as in A in normocapnic $\left(5 \% \mathrm{CO}_{2}\right)$ and hypercapnic $\left(15 \% \mathrm{CO}_{2}\right) \mathrm{SNB}$ solution. Note that hypercapnia reversibly decreased the firing rate of this LC neuron that projects to the cNTS even in the presence of SNB solution. (C) Summary of the effect of hypercapnia in aCSF on firing rate ( $\mathrm{Hz}$ : action potentials per s) in three rhodamine-labeled LC neurons. (D) The reduction in firing rate by hypercapnia was maintained in SNB solution in the same rhodamine-labeled LC neuron shown in B. 


\section{DISCUSSION}

In the present study, we used retrograde labeling in neonatal rat pups to investigate afferent projections from a variety of brain regions to two chemosensitive areas, the LC and the cNTS (Nattie and Li, 2009; Nattie, 2011). In addition, we have begun to characterize the functional connections between these two regions using electrophysiological techniques. Among the main findings, there are considerable anatomical connections between numerous chemosensitive sites and at least some of the connections appear to be capable of transmitting chemosensitive information in neonates as young as P7-P10. These findings indicate that the central chemosensitive network is likely to be both anatomically and functionally complex, even in neonatal rats. Our data also suggest that pathways believed to be involved in the consolidation of aversive and object recognition memories (Clayton and Williams, 2000; Mello-Carpes and Izquierdo, 2013) may be already present in neonatal rats.

\section{Anatomy results}

Afferent projections to the LC. Based on our findings (Figs. 1 and 4 and Table 1), LC neurons in neonatal rats receive afferent projections from many regions involved in cardiorespiratory and autonomic control including dHyp, IHyp, PAG, cLC, SubC, PB, KF, A5, Amb/RVL/CVL, Pre-Bötz/Bötz, cNTS, MR and RTN. The results of the present study in neonatal rats agree in general with a previous study by Cedarbaum and Aghajanian (1978) performed in adult rats with horseradish peroxidase (HRP) injected into LC. These authors showed a similar retrograde labeling pattern in hypothalamus, PB and NTS. Also, other anatomical studies using adult rats have described afferents to the LC from neurons of the PB (Luppi et al., 1995), hypothalamic areas (Conrad and Pfaff, 1976a,b; Saper et al., 1976; Swanson, 1976, 1977; Swanson and Cowan, 1977; Luppi et al., 1995; Lee et al., 2005; Reyes et al., 2005), KF (Luppi et al., 1995), PAG (Ennis et al., 1991; Van Bockstaele and Aston-Jones, 1992; Luppi et al., 1995; Odeh and Antal, 2001; Lee et al., 2005) and MR (Sim and Joseph, 1992).

In relation to PAG, it is important to mention that the retrograde labeling observed in the $\mathrm{LC}+$ group was concentrated on the lateral $(37 \%)$ and ventrolateral $(47 \%)$ region. PAG is divided into four longitudinal columns (dorsomedial, dorsolateral, lateral, and ventrolateral) that are proposed to have differing functions (Carrive and Bandler, 1991; Bandler et al., 1991). Our findings are in agreement with other studies that showed cell bodies of neurons from adults projecting from the PAG to the LC are most concentrated within the ventrolateral column (Morgane and Jacobs, 1979; Mantyh, 1983; Aston-Jones et al., 1991; Luppi et al., 1995; Bajic and Proudfit, 1999; Odeh and Antal, 2001). Likewise, neurons in the ventrolateral column of the PAG also strongly project to the peri-LC (Aston-Jones et al., 1991; Luppi et al., 1995; Bajic and Proudfit, 1999).
We also observed a strong labeling (63\%) in the raphé pallidus. These projections, though sparse, have been confirmed through anterograde methods in adult cats (Bobillier et al., 1976) and rats (Sim and Joseph, 1992) and also through retrograde methods in adult rats (Cedarbaum and Aghajanian, 1978; Morgane and Jacobs, 1979; Luppi et al., 1995). Raphé pallidus is involved in respiratory control in mammals and has excitatory modulation of ventilation (Lalley, 1986; Cao et al., $2006 a, b)$. Neurotracing studies revealed that this nucleus projects to spinal phrenic motor neurons (Dobbins and Feldman, 1994; Hosogai et al., 1998) as well as medullary phrenic pre-motor neurons (Smith et al., 1989; Lindsey et al., 1994; Song et al., 2001).

Regarding NTS, connections from the cNTS to the LC have been demonstrated through anterograde labeling studies (Van Bockstaele et al., 1999) as well as retrograde labeling studies, both in adult rats (Cedarbaum and Aghajanian, 1978; Clavier, 1979; Aston-Jones et al., 1991; Luppi et al., 1995; Rampon et al., 1999). Taken together, these results indicate that afferent projections to LC neurons in neonatal and adult rats are similar and involve numerous regions putatively related to the central chemosensitive network.

It is well known that the dendrites of LC neurons ramify heavily and selectively in the rostromedial part of SubC (Aston-Jones et al., 1991; Van Bockstaele et al., 2001). The innervations of dendrites in peri-LC areas may have a significant influence on the activity of LC neurons because some studies have shown that LC processes may interact directly via electrotonic coupling (Williams et al., 1991; Huang et al., 1997; Dean et al., 1997; Veznedaroglu et al., 1998). This could explain why the peri-LC injected group shows a similar pattern of retrograde labeling as the $\mathrm{LC}+$ group (Table 1). Regardless it is clear that the LC is receiving considerable afferent input from regions involved in the central chemosensitive network, which could account for the ability of serotonergic nerve endings (perhaps from the raphe) to modulate the activity of LC neurons (Cedarbaum and Aghajanian, 1978; Van Bockstaele, 2000) and is consistent with LC neurons exhibiting a level of activity that is modulated at respiratory frequency (Oyamada et al., 1998).

Afferent projections to the cNTS. When rhodamine was injected into the cNTS, the retrograde labeling pattern was smaller compared to LC injections. Further, fewer projections to cNTS were found compared with the LC afferents (Table 1).

Similar to LC, the cNTS neurons from neonatal rats receive afferent projections from regions involved in cardiorespiratory and autonomic control (Figs. 2 and 6 and Table 1), including dHyp, IHyp, PAG, LC, SubC, KF, A5, Amb/RVL/CVL, Pre-Bötz/ Bötz, MR and RTN. Afferent projections to the cNTS have previously been observed from the ventral medullary respiratory group, and specifically from the RVL, Bötz and Pre-Bötz (Tan et al., 2010; Alheid et al., 2011). In addition, projections from hypothalamus to cNTS have also been shown (Van der Kooy et al., 1984). Like the LC, most projections 
to the cNTS observed in adulthood are already established in newborns.

The numerous projections from the ventral medullary respiratory group, including $\mathrm{Amb} / \mathrm{RVL} / \mathrm{CVL}$, Pre-Bötz as well as the RTN strongly suggest that the neonate cNTS is involved in breathing regulation. In fact, cNTS has been suggested to be a site for integration of respiratory, cardiovascular and gastroesophageal systems that work together to eliminate $\mathrm{CO}_{2}$ during acute and chronic respiratory acidosis to restore $\mathrm{pH}$ homeostasis (Dean and Putnam, 2010).

The cNTS Miss group showed little to no retrograde labeling compared to the group where rhodamine injections were clearly in the cNTS (cNTS +) (Table 1), which shows the specificity of the afferent projections for the cNTS.

Functional connections between the cNTS and LC. Considerable anatomical connections among various brainstem regions were found that are believed to be a part of the central chemosensitive network. Since these regions are not only involved in breathing control, it is likely that some of the anatomical connections are involved in other physiological functions. For instance, it is well known that the control of breathing is state dependent, changing with sleep or anesthesia (Nattie, 2001; Kuwaki, 2010). Orexin has been proposed to contribute to state-dependent control of ventilation and orexinergic neurons are located in the hypothalamus, mainly in the IHyp and dHyp (Kuwaki et al., 2008; Kuwaki, 2010; Li and Nattie, 2014; Vicente et al., 2016). It was found in the present study that neurons from these regions project to the cNTS and the LC (Table 1). It is thus possible that some of the afferent projections from the hypothalamus to the cNTS and the LC, observed in the current study, represent orexinergic neurons that participate in state-dependent control of breathing. This would also imply that this state-dependent control might be well developed within the neonatal period.

It is also known that the NTS plays a key role in the consolidation of memories associated with object recognition and aversive stimuli. The key finding is that stimulation of the NTS with noradrenergic (GarciaMedina and Miranda, 2013), adrenergic agents (Clayton and Williams, 2000) or glucocorticoids (Roozendaal et al., 1999) facilitated the consolidation of aversive or object recognition memories. It appears that activation of the NTS works by increasing the release of norepinephrine in the basolateral amygdala. There have been numerous suggestions as to the anatomical neural pathways by which activation of NTS results in adrenergic activation of the basolateral amygdala. There appear to be direct activation pathways from the NTS to the amygdala and at least some of the neurons projecting from the NTS to the amygdala terminate on the basolateral amygdala (Roozendaal et al., 1999; Clayton and Williams, 2000; Garcia-Medina and Miranda, 2013). It is also known that LC neurons project to the amygdala and it has been proposed that activation of the NTS may work through activation of the LC (Clayton and Williams, 2000;
Garcia-Medina and Miranda, 2013). Finally, a more complicated pathway from the NTS to the amygdala has been proposed. In this pathway, activation of the NTS is proposed to send projections to the Paragigantocellularis nucleus, which activates the LC leading to release of noradrenergic agents into the basolateral amygdala (Clayton and Williams, 2000; Mello-Carpes and Izquierdo, 2013).

Our present findings did not enable us to confirm the full connectedness among the NTS, Paragigantocellularis nucleus, the LC and the basolateral amygdala that has been proposed from work in adults (Clayton and Williams, 2000; Mello-Carpes and Izquierdo, 2013), however we did find connections between the NTS and the LC directly, as previously suggested (Clayton and Williams, 2000; Garcia-Medina and Miranda, 2013). It may be that this pathway can play an important part in memory consolidation or it may represent a simpler pathway used in young animals (neonates and juveniles) to consolidate memory. Finally, we report here that exposure of certain cNTS neurons to $15 \% \mathrm{CO}_{2}$ results in activation of those neurons. It is likely that this stimulus, which would undoubtedly be aversive, can activate the CNTS and through whatever pathway, this could serve to consolidate that aversive memory. In this line of reasoning, it may be that consolidation of aversive stimuli may be present at a very early stage of development to foster the survival of the organism whereas memories like object recognition may not be consolidated until the animal is considerably older (Nirogi et al., 2012).

Our speculation here will require further testing of the development of memory consolidation and the establishment of specific neuronal connections between critical brain regions associated with memory consolidation. We believe that our preparation as described here would be most useful for such studies.

Anatomical analysis - considerations. The present results on the anatomical connections between various chemosensitive regions agree with a previous work performed in adult rats; however, there is no study performed in newborn rats to compare with our findings, which makes our results unique. Nevertheless, it is important to consider some points about our anatomical analysis.

Some caution has to be taken when inferring afferent projections from relatively small, highly localized areas. For instance, the RTN involves a rather localized region of chemosensitive neurons near the facial nucleus (Mulkey et al., 2004). While in the present study some retrograde labeling was observed near to the facial nucleus, other data from Rosin et al. (2006) contradict our conclusion that RTN neurons project to the LC, although these authors found retrograde labeling from the SubC region, concluding that RTN neurons do not project directly to the LC. Another important point to be considered is that contradictory results come from adult rats. It has been suggested that ventilatory responses to $\mathrm{CO}_{2}$ change during neonatal development (Stunden et al., 2001; Putnam et al., 2005; Davis et al., 2006). This raises the possibility that the same could happen with brainstem interconnections. 
Thus, RTN neurons may project to the LC during neonatal development but those projections could be lost in adults.

A similar concern applies to the very small pre-Bötz and Bötz regions where anterograde labeling studies in adult rats did not indicate projections to the LC (Ellenberger and Feldman, 1994). It could be that projections from these regions to the LC exist in neonates but not in adults but such a conclusion would require further study.

\section{Electrophysiology}

The question arises as to whether some of the afferent connections between chemosensitive regions of the brainstem directly carry chemosensitive information from one area to another. This would most likely take the form of intrinsically chemosensitive neurons from one region projecting to another chemosensitive region. The ventilatory response to hypercapnia is largely mediated by $\mathrm{CO}_{2}$-sensitive neurons in the brainstem, referred to as central chemosensitive neurons (Coates et al., 1993; Nattie, 2001; Scheid et al., 2001; Putnam et al., 2004; Ritucci et al., 2005). In the present study, to verify if a neuron was chemosensitive we tested if the firing rate of the neuron was altered by changes in $\mathrm{CO}_{2} / \mathrm{H}^{+}$(Figs. 9 and 10). Also, we verified if chemosensitive neurons were intrinsically responsive to changes of $\mathrm{CO}_{2} / \mathrm{H}^{+}$by exposing neurons to altered levels of $\mathrm{CO}_{2} / \mathrm{H}^{+}$in the presence of synaptic block media (Figs. 9 and 10).

Interestingly, some neurons that project from the cNTS to the LC and from the LC to the cNTS had altered firing rate responses to hypercapnic acidosis that persisted in the presence of SNB, suggesting that these neurons are intrinsically responsive to changes in $\mathrm{CO}_{2} / \mathrm{H}^{+}$. In older neonates (Conrad et al., 2009) and adults (Nichols et al., 2009) $45-50 \%$ of the neurons from the cNTS have firing rates that are increased by hypercapnic acidosis, while only $5-10 \%$ have firing rates that are inhibited by hypercapnic acidosis. In neurons from the LC of older neonates, between $20 \%$ and $40 \%$ of the neurons have firing rates that are intrinsically increased by hypercapnic acidosis (Nichols et al., 2008; Gargaglioni et al., 2010) with no reported neurons that are inhibited by hypercapnic acidosis. In our study, of five cNTS neurons patched that project to the LC, four had firing rates increased by hypercapnia. These findings suggest that a high percentage of cNTS neurons that have afferent projections to the LC are intrinsically chemosensitive. Although LC neurons are predominantly activated by $\mathrm{CO}_{2}$ (Filosa et al., 2002), in the present study we found that two of the three LC neurons with afferent projections to the CNTS had firing rates that were inhibited by hypercapnia. In the past, LC neurons that were inhibited by hypercapnic acidosis were occasionally seen but the percentage was so low that such inhibited neurons have never been reported (Putnam-unpublished data). It was reported that epinephrine and norepinephrine both inhibit the activity of cNTS neurons (Feldman and Moises, 1987), so inhibition of retrogradely loaded LC neurons by hypercapnia may increase cNTS neuronal activity or response to hypercapnia by reducing epinephrine and norepinephrine release in cNTS from the terminals of these LC neurons. Thus, these findings suggest that highly specialized LC neurons are the ones with afferent projections to the cNTS.

Based on these functional measurements, we assume that upon exposure to hypercapnia cNTS neurons provide excitatory input to the LC (+? in Fig. 11) while LC neurons provide inhibitory input to the cNTS $(-$ ? in Fig. 11). These conclusions are based on the assumption that both types of neurons that were studied have an excitatory neurotransmitter phenotype. We also do not currently know the relative contribution to central chemosensitivity provided by different chemosensitive regions. One way to consider relative contribution is by the sensitivity to acid stimuli from different regions. Using the same techniques in the same laboratory, we know that the $\mathrm{Cl}$ of chemosensitive neurons (hypercapnic stimulus of $10 \% \mathrm{CO}_{2}$ ) from the RTN of neonatal rats (Ritucci et al., 2005$)$ is larger ( 300\%) than the values of $150-175 \%$ reported for the $\mathrm{Cl}$ of chemosensitive neurons (hypercapnic stimulus of $15 \% \mathrm{CO}_{2}$ ) from the cNTS of neonatal rats (Conrad et al., 2009). Interestingly, the chemosensitive response of LC neurons appears to change with development. In LC neurons from young neonates $(<P 10)$ the $\mathrm{Cl}$ is about $235 \%$ but in $\mathrm{LC}$ neurons from older neonates ( $>$ P10) it is considerably less, around $125 \%$ (Gargaglioni et al., 2010). For neurons from many other chemosensitive regions the $\mathrm{Cl}$ lies between the values of $125-300 \%$ (Putnam et al., 2004). It will remain for future work to fully define how neurons

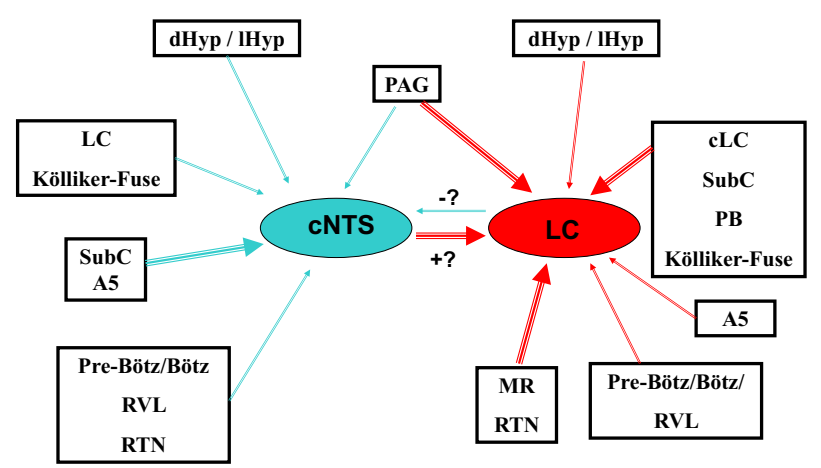

Fig. 11. Schematic diagram of the interconnections among several putative chemosensitive regions of the brainstem, based on our retrograde labeling data. Red arrows indicate afferent projections to the LC while blue arrows represent afferent projections to the CNTS. Thicker arrows indicate stronger retrograde labeling and thus more projecting neurons while thinner arrows represent weaker labeling and thus fewer projecting neurons. The blue arrow connecting LC to cNTS is marked with a "-?" to indicate that this connection may be inhibitory (based on the electrophysiology data) but that this is uncertain until the predominant neurotransmitter of these neurons is known. The red arrow connecting cNTS to LC is marked with a "+?" to indicate that this connection may be excitatory (based on the electrophysiology data) but that this is uncertain until the predominant neurotransmitter of these neurons is known. cNTS, caudal region of the nucleus tractus solitarius near the area postrema; contralateral LC, locus coeruleus on the side opposite the site of injection; $\mathrm{dHyp} /$ IHyp, dorsomedial and lateral hypothalamus; LC, locus coeruleus; MR, medullary raphe; PAG, periaqueductal gray; PB, parabrachial nucleus; RTN, retrotrapezoid nucleus; SubC, subcoeruleus region; Pre-Bötz/Bötz:, Pre-Bötzinger complex and Bötzinger complex; RVL, rostroventrolateral reticular nucleus. 
from various chemosensitive regions contribute to the central chemosensitive network.

Nevertheless, it is clear that the anatomical connections between the cNTS and the LC have the potential to carry functional information about changes in the levels of $\mathrm{CO}_{2} / \mathrm{H}^{+}$. This has recently been confirmed for another part of the respiratory network, where cNTS neurons that are responsive to a similar level of acidosis as used in our study have been shown to project to the ventral respiratory column (Huda et al., 2012). Thus, the sharing of chemosensitive information among various regions of the central chemosensitive network may be widespread.

\section{Summary - interconnections among chemosensitive brain regions}

Fig. 11 shows a schematic representation of the interconnections among several putative chemosensitive regions of the brain in neonatal rats, based on the retrograde labeling data found in the present study. The regions dHyp/lHyp, PAG, cLC, SubC, KF, A5, Pre-Bötz, Bötz, RVL, cNTS, MR and RTN showed potential projections to the cNTS (blue arrows) or to the LC (red arrows). The thicker arrows indicate higher numbers of neurons interconnecting the regions and the thin arrows indicate fewer neurons making interconnections between the regions (data based on Table 1). Most of the neurons that project from cNTS to LC were stimulated by hypercapnia Fig. 9), so it is assumed that these projections are excitatory (+?), whereas some of the neurons that project from LC to cNTS were inhibited by hypercapnia (Fig. 10), suggesting that some of these projections are inhibitory (-?).

\section{Significance and perspectives}

Our findings clearly indicate that these anatomical connections are present in rats 14-20 days after birth and most likely at least as early as $7-10$ days post birth. The rhodamine bead injections were made in neonatal rats aged P7-P10 and we presume that the projections were intact at that age in order for retrograde labeling to occur. We found that it required an additional 7-10 days to see retrogradely labeled neurons with somas that are clearly labeled. We assume that this time reflects the long transport distance required in these neurons and not additional time for projections to be established. Further, at least for cNTS neurons projecting to the LC, the pattern of sensitivity of firing rate to hypercapnia in these neurons is unchanged in neurons from rats aged P1 through the entire neonatal period (Conrad et al., 2009) and into adulthood (Nichols et al., 2009). Based on these findings, we suggest that both anatomical and electrophysiological aspects of the respiratory network are developed in neonatal rats as early as the first week of life. It is possible that the network is established even earlier but we were unable to perform retrograde labeling studies in neonatal rats younger than $\mathrm{P} 7$ so it will be difficult to determine how early the network is established with retrograde labeling techniques.
The data presented here (Figs. 9 and 10) as well as those of others (Huda et al., 2012) suggest that there is direct exchange of chemosensitive information between central chemosensitive areas through the extensive anatomical couplings that were observed here (Fig. 11). Thus the alteration of firing rate by hypercapnic acidosis in chemosensitive neurons from one area can directly result in altered firing rate in neurons from other chemosensitive regions. These finding can help explain an interesting phenomenon. It has long been known that when multiple chemosensitive areas are individually focally acidified, the sum of increased ventilation of all the individual areas can exceed the increased ventilation induced by acidifying the whole brain with increased inspired $\mathrm{CO}_{2}$ (Coates et al., 1993; Putnam et al., 2004). This would be explained if focally acidifying one chemosensitive region of the brainstem could activate other regions through excitatory projections. If this is occurring, it will also mean that caution must be used when interpreting focal acidosis experiments as indicating the contribution of just the acidified region to the hypercapnic ventilatory response. It is likely that such experiments could overestimate the contribution of a given area because acidification of that area may result in stimulation of other, non-acidified chemosensitive regions through the respiratory network.

Finally, our work suggests several research challenges for the future. The major challenge is to describe the neurotransmitter phenotype of the afferent projecting neurons between various chemosensitive regions to determine the proportion of neurons that are modulatory (e.g. positive for serotonin or orexin), that are excitatory and that are inhibitory. Also, more electrophysiological work needs to be done to determine the extent to which intrinsically chemosensitive neurons project between different chemosensitive regions. Finally, these electrophysiological studies need to be combined with immunohistochemical studies to determine what neurotransmitters are released by the projecting chemosensitive neurons in order to fully describe exactly what information is being exchanged between different chemosensitive regions in response to hypercapnic acidosis.

As stated above, our work also suggests future directions for the study of the role of various brainstem regions in the development of memory consolidation and whether differences may exist in the development of aversive memories as opposed to other types of memories.

\section{CONCLUSIONS}

The main conclusion from this study is that, in neonatal rats, there are extensive anatomical connections between different regions shown to be involved in central chemosensitivity (and possibly involved in the consolidation of memory). There are no other studies in the literature about the respiratory control network in neonates with which to compare our results but our findings agree with other studies 
suggesting extensive inter-connections among many of these regions in adult rats. Further we demonstrated that at least some of these connections between respiratory control areas involve the exchange of chemosensitive information. Our work will contribute to the understanding of the development of the respiratory control network.

\section{AUTHOR CONTRIBUTIONS}

L.T.L., L.G.A.P., K.Y.L., A.N.I. and C.D.G. collected and analyzed data. L.T.L., L.G.A.P., L.G. and R.W.P. wrote and edited the manuscript.

\section{CONFLICT OF INTEREST}

There are no conflicts of interest.

Acknowledgments-This work was supported by American Heart Association Great Rivers Affiliate Predoctoral Fellowship (ANI), NIH R01-HL-56683 (RWP), Research Challenge Augmentation Grant from Wright State University (RWP), São Paulo Research Foundation (FAPESP), National Council for Scientific and Technological Development (CNPq, Brazil) and a FAPESP Fellowship Grants: 2008/57754-9 (to L.T.L.) and 2010/06210-9 (to L.G.A.P).

\section{REFERENCES}

Alheid GF, Jiao W, Mccrimmon DR (2011) Caudal nuclei of the rat nucleus of the solitary tract differentially innervate respiratory compartments within the ventrolateral medulla. Neuroscience 190:207-227.

Aston-Jones G, Shipley MT, Chouvet G, Ennis M, Van Bockstaele E, Pieribone V, Shiekhattar R, Akaoka H, Drolet G, Astier B, Charléty P, Valentino RJ, Williams JT (1991) Afferent regulation of locus coeruleus neurons: anatomy, physiology and pharmacology. Prog Brain Res 88:47-75.

Bajic D, Proudfit HK (1999) Projections of neurons in the periaqueductal gray to pontine and medullary catecholamine cell groups involved in the modulation of nociception. J Comp Neurol 405:359-379.

Bandler R, Carrive P, Zhang SP (1991) Integration of somatic and autonomic reactions within the midbrain periaqueductal grey: viscerotopic, somatotopic and functional organization. Prog Brain Res 87:269-305.

Barraco RA, Janusz CA, Schoener EP, Simpson LL (1990) Cardiorespiratory function is altered by picomole injections of $5^{\prime}$ $\mathrm{N}$-ethylcarboxamidoadenosine into the nucleus tractus solitarius of rats. Brain Res 507:234-246.

Berger AJ, Cooney KA (1982) Ventilatory effects of kainic acid injection of the ventrolateral solitary nucleus. J Appl Physiol 52:131-140.

Biancardi V, Bícego KC, Almeida MC, Gargaglioni LH (2008) Locus coeruleus noradrenergic neurons and $\mathrm{CO}_{2}$ drive to breathing. Pflügers Arch 55:119-128.

Bobillier P, Seguin S, Petitjean F, Salvert D, Touret M, Jouvet M (1976) The raphe nuclei of the cat brain stem: a topographical atlas of their efferent projections as revealed by autoradiography. Brain Res 113:449-486.

Bonham AC, McCrimmon DR (1990) Neurones in a discrete region of the nucleus tractus solitarius are required for the Breuer-Hering reflex in rat. J Physiol 427:261-280.

Cao Y, Fujito Y, Matsuyama K, Aoki M (2006a) Effects of electrical stimulation of the medullary raphe nuclei on respiratory movement in rats. J Comp Physiol A Neuroethol Sens Neural Behav Physiol 192:497-505.
Cao Y, Matsuyama K, Fujito Y, Aoki M (2006b) Involvement of medullary GABAergic and serotonergic raphe neurons in respiratory control: electrophysiological and immunohistochemical studies in rats. Neurosci Res 56:322-331.

Carrive P, Bandler R (1991) Viscerotopic organization of neurons subserving hypotensive reactions within the midbrain periaqueductal grey: a correlative functional and anatomical study. Brain Res 541:206-215.

Cedarbaum JM, Aghajanian GK (1978) Afferent projections to the rat locus coeruleus as determined by a retrograde tracing technique. J Comp Neurol 178:1-16.

Clavier RM (1979) Afferent projections to the self-stimulation regions of the dorsal pons, including the locus coeruleus, in the rat as demonstrated by the horseradish peroxidase technique. Brain Res Bull 4:497-504.

Clayton EC, Williams CL (2000) Adrenergic activation of the nucleus tractus solitarius potentiates amygdala norepinephrine release and enhances retention performance in emotionally arousing and spatial memory tasks. Behav Brain Res 112:151-158.

Coates EL, Li A, Nattie EE (1993) Widespread sites of brain stem ventilatory chemoreceptors. J Appl Physiol 75:5-14.

Conrad LC, Pfaff DW (1976a) Efferents from medial basal forebrain and hypothalamus in the rat. I. An autoradiographic study of the medial preoptic area. J Comp Neurol 169:185-219.

Conrad LC, Pfaff DW (1976b) Efferents from medial basal forebrain and hypothalamus in the rat. II. An autoradiographic study of the anterior hypothalamus. J Comp Neurol 169:221-261.

Conrad SC, Nichols NL, Ritucci NA, Dean JB, Putnam RW (2009) Development of chemosensitivity in neurons from the nucleus tractus solitarii (NTS) of neonatal rats. Respir Physiol Neurobiol 166:4-12.

Davis SE, Solhied G, Castillo M, Dwinell M, Brozoski D, Forster HV (2006) Postnatal developmental changes in $\mathrm{CO}_{2}$ sensitivity in rats. J Appl Physiol 101:1097-1103.

Dean JB, Putnam RW (2010) The caudal solitary complex is a site of central $\mathrm{CO}_{2}$ chemoreception and integration of multiple systems that regulate expired $\mathrm{CO}_{2}$. Respir Physiol Neurobiol 173:274-287.

Dean JB, Lawing WL, Millhorn DE (1989) $\mathrm{CO}_{2}$ decreases membrane conductance and depolarizes neurons in the nucleus tractus solitarii. Exp Brain Res 76:656-661.

Dean JB, Bayliss DA, Erickson JT, Lawing WL, Millhorn DE (1990) The depolarization and stimulation of neurons in nucleus tractus solitarii by carbon dioxide does not require chemical synaptic input. Neuroscience 36:207-216.

Dean JB, Huang RQ, Erlichman JS, Southard TL, Hellard DT (1997) Cell-cell coupling occurs in dorsal medullary neurons after minimizing anatomical-coupling artifacts. Neuroscience $80: 21-40$.

Dobbins EG, Feldman JL (1994) Brainstem network controlling descending drive to phrenic motoneurons in rat. $\mathrm{J}$ Comp Neurol 347:64-86.

Donoghue S, Felder RB, Jordan D, Spyer KM (1984) The central projections of carotid baroreceptors and chemoreceptors in the cat: a neurophysiological study. J Physiol 347:397-409.

Elam M, Yao T, Thorén P, Svensson TH (1981) Hypercapnia and hypoxia: chemoreceptor-mediated control of locus coeruleus neurons and splanchnic, sympathetic nerves. Brain Res 222:373-381.

Ellenberger HH, Feldman JL (1994) Origins of excitatory drive within the respiratory network: anatomical localization. NeuroReport 5:1933-1936.

Ennis M, Behbehani M, Shipley MT, Van Bockstaele EJ, AstonJones G (1991) Projections from the periaqueductal gray to the rostromedial pericoerulear region and nucleus locus coeruleus: anatomic and physiologic studies. J Comp Neurol 306:480-494.

Favero MT, Takakura AC, de Paula PM, Colombari E, Menani JV, Moreira TS (2011) Chemosensory control by commisural nucleus of the solitary tract in rats. Respir Physiol Neurobiol 179:227-234.

Feldman PD, Moises HC (1987) Adrenergic responses of baroreceptive cells in the nucleus tractus solitarii of the rat: a microiontophoretic study. Brain Res 420:351-361. 
Filosa JA, Dean JB, Putnam RW (2002) Role of intracellular and extracellular $\mathrm{pH}$ in the chemosensitive response of rat locus coeruleus neurones. J Physiol 541:493-509.

Garcia-Medina NE, Miranda MI (2013) Nucleus of the solitary tract chemical stimulation induces extracellular norepinephrine release in the lateral and basolateral amygdala. Brain Stimul 6:198-201.

Gargaglioni LH, Hartzler LK, Putnam RW (2010) The locus coeruleus and central chemosensitivity. Respir Physiol Neurobiol 173:264-273.

Greer JJ (2012) Control of breathing activity in the fetus and newborn. Comp Physiol 2:1873-1888.

Hartzler LK, Dean JB, Putnam RW (2008) The chemosensitive response of neurons from the locus coeruleus (LC) to hypercapnic acidosis with clamped intracellular pH. Adv Exp Med Biol 605:333-337.

Hodges MR, Richerson GB (2010) The role of medullary serotonin (5HT) neurons in respiratory control: contributions to eupneic ventilation, $\mathrm{CO}_{2}$ chemoreception, and thermoregulation. J Appl Physiol 108:1425-1432.

Hosogai M, Matsuo S, Sibahara T, Kawai Y (1998) Projection of respiratory neurons in rat medullary raphé nuclei to the phrenic nucleus. Respir Physiol 112:37-50.

Huang RQ, Erlichman JS, Dean JB (1997) Cell-cell coupling between $\mathrm{CO}_{2}$-excited neurons in the dorsal medulla oblongata. Neuroscience 80:41-57.

Huda R, Pollema-Mays SL, Chang Z, Alheid GF, McCrimmon DR, Martina M (2012) ASIC channels contribute to chemosensitivity of breathing-related NTS neurones. J Physiol 590:4761-4775.

Koshiya N, Guyenet PG (1996) NTS neurons with carotid chemoreceptor inputs arborize in the rostral ventrolateral medulla. Am J Physiol 270:R1273-R1278.

Kuwaki T (2010) Hypothalamic modulation of breathing. Adv Exp Med Biol 669:243-247.

Kuwaki T, Zhang W, Nakamura A, Deng BS (2008) Emotional and state-dependent modification of cardiorespiratory function: role of orexinergic neurons. Auton Neurosci 142:11-16.

Lalley PM (1986) Responses of phrenic motoneurons of the cat to stimulation of medullary raphé nuclei. J Physiol 380:349-371.

Lee HS, Kim MA, Waterhouse BD (2005) Retrograde double-labeling study of common afferent projections to the dorsal raphe and the nuclear core of the locus coeruleus in the rat. J Comp Neurol 481:179-193.

Li A, Nattie E (2006) Catecholamine neurones in rats modulate sleep breathing, central chemoreception and breathing variability. J Physiol 570:385-396.

Li A, Nattie E (2014) Orexin, cardio-respiratory function, and hypertension. Front Neurosci 8:22.

Li K-Y, Putnam RW (2013) Transient outwardly rectifying A currents are involved in the firing rate response to altered $\mathrm{CO}_{2}$ in chemosensitive locus coeruleus neurons from neonatal rats. Am J Physiol 305:R780-R792.

Lindsey BG, Segers LS, Morris KF, Hernandez YM, Saporta S, Shannon R (1994) Distributed actions and dynamic associations in respiratory-related neuronal assemblies of the ventrolateral medulla and brain stem midline: evidence from spike train analysis. J Europhysiol 72:1830-1851.

Luppi PH, Aston-Jones G, Akaoka H, Chouvet G, Jouvet M (1995) Afferent projections to the rat locus coeruleus demonstrated by retrograde and anterograde tracing with cholera-toxin B subunit and Phaseolus vulgaris leucoagglutinin. Neuroscience 65:119-160.

Mantyh PW (1983) Connections of midbrain periaqueductal gray in the monkey. I. Ascending efferent projections. J Neurophysiol 49:567-581.

McGaugh JL (2000) Memory - a century of consolidation. Science 287:248-251.

Mello-Carpes PB, Izquierdo I (2013) The nucleus of the solitary tract $\rightarrow$ nucleus Paragigantocellularis $\rightarrow$ locus coeruleus $\rightarrow$ CA1 region of dorsal hippocampus pathway is important for consolidation of object recognition memory. Neurobiol Learn Mem 100:56-63.
Mifflin SW (1992) Arterial chemoreceptor input to nucleus tractus solitarius. Am J Physiol 263:R368-R375.

Morgane PJ, Jacobs MS (1979) Raphe projections to the locus coeruleus in the rat. Brain Res Bull 4:519-534.

Mortola JP (2001) Respiratory physiology of newborn mammals: a comparative perspective. Baltimore: The Johns Hopkins University Press.

Mulkey DK, Stornetta RL, Weston MC, Simmons JR, Parker A, Bayliss DA, Guyenet PG (2004) Respiratory control by ventral surface chemoreceptor neurons in rats. Nat Neurosci 7:1360-1369.

Nattie E (1999) $\mathrm{CO}_{2}$, brainstem chemoreceptors and breathing. Prog Neurobiol 59:299-331.

Nattie EE (2001) Central chemosensitivity, sleep, and wakefulness. Respir Physiol 129:257-268.

Nattie E (2011) Julius H. Comroe, Jr., distinguished lecture: central chemoreception: then. . . and now. J Appl Physiol 110:1-8.

Nattie EE, Li A (2002) $\mathrm{CO}_{2}$ dialysis in nucleus tractus solitarius region of rat increases ventilation in sleep and wakefulness. J Appl Physiol 92:2119-2130.

Nattie EE, Li A (2008) Muscimol dialysis into the caudal aspect of the nucleus tractus solitarii of conscious rats inhibits chemoreception. Respir Physiol Neurobiol 164:394-400.

Nattie E, Li A (2009) Central chemoreception is a complex system function that involves multiple brain stem sites. J Appl Physiol 106:1464-1466.

Nichols NL, Hartzler LK, Conrad SC, Dean JB, Putnam RW (2008) Intrinsic chemosensitivity of individual nucleus tractus solitarius (NTS) and locus coeruleus (LC) neurons from neonatal rats. Adv Exp Med Biol 605:348-352.

Nichols NL, Mulkey DK, Wilkinson KA, Powell FL, Dean JB, Putnam RW (2009) Characterization of the chemosensitive response of individual solitary complex (SC) neurons from adult rats. Am J Physiol 296:R763-R773.

Nirogi R, Abraham R, Jayarajan P, Medapati RB, Shanmuganathan D, Kandikere V, Irappanavar S, Saralaya R, Benade V, Bhyrapuneni G, Muddana N (2012) Difference in the norepinephrine levels of experimental and non-experimental rats with age in the object recognition task. Brain Res 1453:40-45.

Odeh F, Antal M (2001) The projections of the midbrain periaqueductal grey to the pons and medulla oblongata in rats. Eur J Neurosci 14:1275-1286.

Oyamada Y, Ballantyne D, Mückenhoff K, Scheid P (1998) Respiration- modulated membrane potential and chemosensitivity of locus coeruleus neurones in the in vitro brainstem-spinal cord of the neonatal rat. J Physiol 513:381-398.

Paxinos G, Watson C (1997). The rat brain in stereotaxic coordinates, vol. 3. San Diego: Academic Press.

Pineda J, Aghajanian GK (1997) Carbon dioxide regulates the tonic activity of locus coeruleus neurons by modulating a proton- and polyamine-sensitive inward rectifier potassium current. Neuroscience 77:723-743.

Putnam RW (2010) $\mathrm{CO}_{2}$ chemoreception in cardiorespiratory control. J Appl Physiol 108:1796-1802.

Putnam RW, Filosa JA, Ritucci NA (2004) Cellular mechanisms involved in $\mathrm{CO}_{2}$ and acid signaling in chemosensitive neurons. Am J Physiol 287:C1493-C1526.

Putnam RW, Conrad SC, Gdovin MJ, Erlichman JS, Leiter JC (2005) Neonatal maturation of the hypercapnic ventilatory response and central neural $\mathrm{CO}_{2}$ chemosensitivity. Respir Physiol Neurobiol 149:165-179.

Rampon C, Peyron C, Gervasoni D, Pow DV, Luppi PH, Fort P (1999) Origins of the glycinergic inputs to the rat locus coeruleus and dorsal raphe nuclei: a study combining retrograde tracing with glycine immunohistochemistry. Eur J Neurosci 11:1058-1066.

Reyes BAS, Valentino RJ, Xu G, Van Bockstaele EJ (2005) Hypothalamic projections to locus coeruleus neurons in rat brain. Eur J Neurosci 22:93-106.

Richerson GB (2004) Serotonergic neurons as carbon dioxide sensors that maintain $\mathrm{pH}$ homeostasis. Nat Rev Neurosci 5:449-461. 
Ritucci NA, Erlichman JS, Leiter JC, Putnam RW (2005) Response of membrane potential and intracellular $\mathrm{pH}$ to hypercapnia in neurons and astrocytes from rat retrotrapezoid nucleus. Am J Physiol 289:R851-R861.

Roozendaal B, Williams CL, McGaugh JL (1999) Gluicocorticoid receptor activation in the rat nucleus of the solitary tract facilitates memory consolidation: involvement of the basolateral amygdala. Eur J Neurosci 11:1317-1323.

Rosin DL, Chang DA, Guyenet PG (2006) Afferent and efferent connections of the rat retrotrapezoid nucleus. J Comp Neurol 499:64-89.

Saper CB, Swanson LW, Cowan WM (1976) The efferent connections of the ventromedial nucleus of the hypothalamus of the rat. J Comp Neurol 169:409-442.

Scheid P, Putnam RW, Dean JB, Ballantyne D (2001) Special issue: central chemosensitivity. Respir Physiol 129:1-278.

Sim LJ, Joseph SA (1992) Efferent projections of the nucleus raphe magnus. Brain Res Bull 28:679-682.

Smith JC, Morrison DE, Ellenberger HH, Otto MR, Feldman JL (1989) Brain stem projections to the major respiratory neuron populations in the medulla of the cat. J Comp Neurol 281:69-96.

Song G, Li Q, Shao FZ (2001) Projections of 5-HTergic fibers to the spinal phrenic nucleus and medullary phrenic premotor neurons in the cat. Sheng Li Xue Bao 53:391-395.

Stunden CE, Filosa JA, Garcia AJ, Dean JB, Putnam RW (2001) Development of in vivo ventilatory and single chemosensitive neuron responses to hypercapnia in rats. Respir Physiol 127:135-155.

Swanson LW (1976) An autoradiographic study of the efferent connections of the preoptic region in the rat. J Comp Neurol 167:227-256.

Swanson LW (1977) Immunohistochemical evidence for a neurophysin-containing autonomic pathway arising in the paraventricular nucleus of the hypothalamus. Brain Res 128:346-353.

Swanson LW, Cowan WM (1977) Autoradiographic studies of the development and connections in the septal area in the rat. In:
DeFrance JF, editor. The septal nuclei, vol. 1. New York: Plenum Press. p. 37-64.

Tan YF, Preston E, Wojtowicz JM (2010) Enhanced post-ischemic neurogenesis in aging rats. Front Neurosci 4:163.

Van Bockstaele EJ (2000) Multiple substrates for serotonergic modulation of rat locus coeruleus neurons and relationships with kainate receptors. Brain Res Bull 51:433-442.

Van Bockstaele EJ, Aston-Jones G (1992) Collateralized projections from neurons in the rostral medulla to the nucleus locus coeruleus, the nucleus of the solitary tract and the periaqueductal gray. Neuroscience 49:653-668.

Van Bockstaele EJ, Peoples J, Telegan P (1999) Efferent projections of the nucleus of the solitary tract to peri-locus coeruleus dendrites in rat brain: evidence for a monosynaptic pathway. J Comp Neruol 412:410-428.

Van Bockstaele EJ, Bajic D, Proudfit H, Valentino RJ (2001) Topographic architecture of stress-related pathways targeting the noradrenergic locus coeruleus. Physiol Behav 73:273-283.

Van der Kooy D, Koda LY, McGinty JF, Gerfen CR, Bloom FE (1984) The organization of projections from the cortex, amygdala, and hypothalamus to the nucleus of the solitary tract in rat. J Comp Neurol 224:1-24.

Veznedaroglu E, Dareshuri-Daou M, Alvarez Maubecin V, Williams JT, Van Bockstaele EJ (1998) Anatomical substrates underlying electrotonic coupling in the rat locus coeruleus. Soc Neurosci Abstr 24:1596.

Vicente MC, Dias MB, Fonseca EM, Bícego KC, Gargaglioni LH (2016) Orexinergic system in the locus coeruleus modulates the $\mathrm{CO}_{2}$ ventilatory response. Pflugers Arch. http://dx.doi.org/ 10.1007/s00424-016-1793-x.

Williams JT, Bobker DH, Harris GC (1991) Synaptic potentials in locus coeruleus neurons in brain slices. Prog Brain Res 88:167-172.

Wong-Riley MT, Liu Q, Gao XP (2013) Peripheral-central chemoreceptor interaction and the significance of a critical period in the development of respiratory control. Respir Physiol Neurobiol 185:156-169. 